RESEARCH PAPER RP1149

\author{
Part of Journal of Research of the National Bureau of Standards, Volume 21, \\ November 1938
}

\title{
EFFECT OF FILLING AND SIZING MATERIALS ON STABILITY OF BOOK PAPERS
}

\author{
By Merle B. Shaw and Martin J. O'Leary
}

\begin{abstract}
The National Bureau of Standards has been carrying on research on the manufacture of papers to obtain information on composition and processes which have an important bearing on the stability of paper. Several publications have been issued as a result of these investigations. Three of them, Research Papers RP372, RP574, and RP794, present data for writing papers. Another Research Paper, RP949, reports the results on experimental unsized papers made from pulps commonly used in book papers. The present publication is an extension of the work on book paper, and relates primarily to the effect of filling and sizing
\end{abstract} variables introduced in manufacture.

The papermaking materials used were representative commercial products. Four types of fillers, including both natural materials and manufactured pigments, were used. They comprised one clay filler; two titanium dioxide pigments; two zinc sulfide pigments; and two calcium carbonates, one precipitated, the other a natural product, water-ground. The sizing agent, rosin soap prepared from rosin and soda ash, was precipitated by means of papermaker's alum, aluminum sulfate. The fibrous materials covered the range of those commonly used in the fine printing papers. They consisted of sulfite pulp and soda pulp of the ordinary book-paper grade; three "purified" wood pulps, produced by special cooking and bleaching treatments to obtain high purity and strength; new rags, the grade known commercially as No. 1 white shirt cuttings; and two grades of old rags, No. 1 old whites and "twos and blues."

Seventy-two experimental papers were manufactured in the Bureau's semicommercial mill. The papers were given extensive physical and chemical tests with particular reference to stability, both before and after an accelerated aging test made by heating the paper for 72 hours at $100^{\circ} \mathrm{C}$.

The strength of the experimental papers decreased with increasing filler content, and was influenced by the amount, not the type, of filler present. There was no pronounced difference in the relative effect of the nonalkaline fillers on sizing. The nonalkaline fillers had less effect than calcium carbonate in reducing the degree of sizing. Although the sizing values of the carbonate-filled papers were not high, the papers were sized sufficiently to be written on with ink and for ordinary printing processes. Maximum clay retention was obtained in the purified wood and the rag papers when the $\mathrm{pH}$ at the head box was approximately 5 , and decreased as the amount of alum was increased. For the sulfite-soda stock, retention of all the nonalkaline fillers increased as alum was increased. The papers containing titanium dioxide, zinc sulfide, or precipitated calcium carbonate pigments had the highest opacity. Preliminary printing tests made on a few of the filled papers indicated satisfactory printing quality.

The rag and purified wood-pulp papers were more stable to the heat test than the sulfite-soda. Nonalkaline fillers had no apparent harmful influence on the stability of any of the papers, and the calcium carbonate pigments had a protective or inhibiting effect in the aging test.

Acidity was an important factor in deterioration. The attack on the cellulose was increased as the amount of alum was increased, in either the unsized or the rosin-sized papers.

The effect of increasing the amount of alum in the beater and then neutralizing part of the alum with sodium carbonate as the stock was being pumped from 
the beater chest to the machine chest was practically the same as having had the final $\mathrm{pH}$ value originally in the beater and maintained throughout the preparation of the stock.

\section{CONTENTS}

I. Introduction

II. Papermaking raw materials

III. Papermaking equipment_._.

IV. Papermaking processes

1. Preparation of rag half stuff

2. Beater and paper-machine operations

V. Testing methods

VI. Analyses of papermaking materials used

1. Fibers . .

VII. Data on papers made

1. Physical and chemical measurements

(a) Clay-filled papers

(1) Sulfite pulp, 50 percent; soda pulp, 50 percent_ 676

(2) Purified wood pulp: $A, 75$ percent; $C, 25$ percent....... 685

(3) Purified wood pulp: $D, 100$ percent...... 686

(4) Rags: No. 1 new whites, 100 percent_..... 686

(5) Rags: No. 1 old whites, 50 percent; twos and blues, 50 percent......... 688

(b) Titanium-dioxide-pigmented papers

(1) Sulfite pulp, 50 percent; soda pulp, 50 percent_ 689

(2) Rags: No. 1 old whites, 50 percent; twos and blues, 50 percent

(c) Zinc-sulfide-pigmented papers. 689

(1) Sulfite pulp, 50 percent; soda pulp, 50 percent_ 690

(2) Rags: No. 1 old whites, 50 percent; twos and blues, 50 percent._. 690

(d) Calcium-carbonate-pigmented papers. 690

(1) Sulfite pulp, 50 percent; soda pulp, 50 percent_ 691

(2) Rags: No. 1 old whites, 50 percent; twos and blues, 50 percent.

2. Printing tests

VIII. Discussion and conclusions

IX. References

\section{INTRODUCTION}

As a part of its program of research relating to the stability of papers used for records, the National Bureau of Standards is making a study of the relation of papermaking materials and processes to the strength, stability, and other properties of book papers. This publication is the fifth in the series planned to include the more important types of fibrous and nonfibrous raw materials commonly used in the manufacture of record papers. Of the preceding publications, three $[1,2,3]^{1}$ related to writing papers and one [4] to fibers commonly found in book papers. The present publication is an extension of the work on book papers and deals primarily with the effect of filling and sizing materials on their stability.

\section{PAPERMAKING RAW MATERIALS}

The fillers selected were representative of several types, both natural materials and manufactured pigments, and were used as supplied by the manufacturers. They comprised one clay; two titanium pigments,

\footnotetext{
1 Figures in brackets indicate the literature references at the end of this paper.
} 
titanium dioxide and a composite of titanium dioxide and barium sulfate; two zinc sulfide pigments, zinc sulfide and a composite of zinc sulfide and barium sulfate; two calcium carbonates, one precipitated, and one water-ground. Fillers are not added to book paper to adulterate it but to improve the printing quality of the sheet. They fill the interstices of the fiber network of the paper thus producing a more even surface, lend softness, and improve the opacity and, generally, the color of the sheet. The number of types of important fillers on the market has increased considerably in the last few years, but little is known about their effect on the permanence of paper. The selection of fillers for the papermaking tests of this study was limited, however, to only the more important types, as a complete study of fillers, as such, was not planned at this time.

The sizing agent was that most generally employed, rosin soap. It was prepared from rosin and soda ash and was precipitated with papermaker's alum, aluminum sulfate.

The fibrous materials employed covered the range of those commonly used in the fine printing papers, and, like the fillers, were obtained from commercial manufacturers. They consisted of sulfite pulp and soda pulp of the ordinary book-paper grade; three "purified" wood pulps produced by special cooking and bleaching treatment to obtain high purity and strength; new rags, the grade known commercially as No. 1 white shirt cuttings; and two grades of old rags, No. 1 old whites and "twos and blues."

\section{PAPERMAKING EQUIPMENT}

The Bureau paper mill is equipped for experimental manufacture of practically all types of paper under conditions which in general simulate those of industrial mills. A complete description and photographs of the equipment may be found in previous publications [5].

\section{PAPERMAKING PROCESSES}

\section{PREPARATION OF RAG HALF STUFF}

The procedure followed in the preparation of the rag "half stuff" (partially pulped rags that have been boiled, washed, drawn out of weave, and bleached) was essentially the same as the general practice in the commercial production of high-grade papers from rags. It was described in a previous publication [3] of the series.

The amount of bleaching powder, containing 35 percent of available chlorine, required to produce the desired degree of whiteness varied with the color of the rags. The amount used, based on the oven-dry weight of the rags, for No. 1 white shirt cuttings was 0.1 to 0.2 percent; for No. 1 old whites, 0.3 percent; and for twos and blues, 1.0 percent.

\section{BEATER AND PAPER-MACHINE OPERATIONS}

To afford comparison of the papers made in the numerous experimental runs it was necessary to follow a uniform procedure for handling the papermaking materials and the paper machine. It was desired that the procedure conform to customary or established general mill practice, but inasmuch as different mills differ widely as 
to the relative time of adding the pulps, fillers, rosin, and alum to the beater, the method used was the one ordinarily followed at the Bureau and previously found to compare favorably with commercial mill methods.

The fillers were mixed with water (made into "slips") and the mixture agitated a fixed length of time and then run through an 80-mesh screen to remove dirt and impurities before being added to the beater.

The procedure followed in furnishing the beater, unless noted otherwise elsewhere in the text, was as follows: The pulps or fibrous materials and the filler slip were put in at the time of furnishing (which required about 15 minutes), and the rosin size was added to the stock 1 hour, and the alum $1 / 2$ hour, before it was dropped to the beater chest. Variation from this procedure might have affected the characteristics of the finished sheet as to bulk, opacity, etc., but it is believed that it did not affect the permanence of the paper, which is the property of primary interest in this study.

The beating procedure was adjusted to the peculiarities of the different papermaking fibers. The paper-machine operations were the same for all runs. The methods of beating and paper-machine operation followed very closely those described in the other publications of this investigation $[1,2,3]$.

The temperature of the stock at the head box of the paper machine was maintained at $90^{\circ} \mathrm{F} \pm 2^{\circ}$.

\section{TESTING METHODS}

All the physical and most of the chemical tests of the pulps and papers were made by the official methods ${ }^{2}$ of the Technical Association of the Pulp and Paper Industry. For the determinations of the amounts of alpha-, beta-, and gamma-cellulose, pentosans, and acidity in the cellulosic materials, the methods used were modifications recently developed at the Bureau [6]. ${ }^{3}$ Although all papers were tested for acidity by the modified method (cold extraction), some of them were tested also by the TAPPI method (hot extraction), and for the latter the values obtained by both methods are reported. For the mill waters - in the beater and the head box-the $\mathrm{pH}$ determinations were made electrometrically, using the quinhydrone method except for the runs with calcium carbonate, for which a glass electrode was used because of the alkalinity.

The relative stability of papers can be judged by determining their chemical characteristics, but in addition it is desirable to subject them to some form of accelerated aging. Therefore, the pertinent physical and chemical tests were applied not only to the original papers but also to samples that had been submitted to an accelerated aging test considered to closely simulate the effects of natural aging. This test is made by heating specimens of the papers for 72 hours at $100^{\circ} \mathrm{C}$ and then conditioning and determining to what extent the paper has been altered in folding endurance, tearing strength, alphacellulose content, and copper number. For details of the accelerated aging test the reader is referred to previous discussions of the method [7].

2 Copies of the methods can be obtained from the Technical Association of the Pulp and Paper Industry,

22 East 42d Street, New York, N. Y.

3 Pentosans and acidity determined by unpublished methods. 


\section{ANALYSES OF PAPERMAKING MATERIALS USED}

\section{FIBERS}

Analyses showing the degree of cellulosic purity of the wood pulps and the rag half stuffs used in the manufacture of the papers are given in table 1. The fibrous material ranged in quality from 74 to 95 percent of alpha-cellulose and from 4.4 to 0.3 in copper number.

TABLE 1.-Chemical test data on fibrous materials used

\begin{tabular}{|c|c|c|c|c|c|c|c|}
\hline Fibrous material & $\begin{array}{l}\text { Alpha- } \\
\text { cellu- } \\
\text { lose } 1\end{array}$ & $\begin{array}{l}\text { Beta- } \\
\text { cellu- } \\
\text { lose } 1\end{array}$ & $\begin{array}{c}\text { Gamma } \\
\text { cellu- } \\
\text { lose } 1\end{array}$ & $\begin{array}{c}\text { Pento- } \\
\text { sans }\end{array}$ & $\begin{array}{l}\text { Copper } \\
\text { number }\end{array}$ & Ash 2 & Resin 2 \\
\hline Sulfite pulp. & $\begin{array}{l}\% \\
82.0\end{array}$ & $\%_{5.8}$ & $\begin{array}{l}\% \\
12.2\end{array}$ & $\%_{5.8}$ & 3.4 & $\begin{array}{l}\% \\
0.1\end{array}$ & $\%_{0.6}$ \\
\hline Soda pulp............. & 74.4 & 21.3 & 4. 3 & 18. 7 & 4. 4 & .3 & .2 \\
\hline Purified wood pulp $A^{3}$ & 91.0 & 4. 2 & 4. 8 & 3.2 & 0.6 & .1 & 2 \\
\hline Purified wood pulp $C^{3}$ & 88.3 & 6.3 & 5.4 & 4. 3 & .8 & .1 & .2 \\
\hline Purified wood pulp $D^{3}$ & 84.2 & 4. 3 & 11.5 & 8. 4 & .6 & .2 & .1 \\
\hline No. 1 new white rags, bleached half stuff. & 94.8 & 4. 7 & 0.5 & -1 & .3 & .1 & \\
\hline No. 1 old white rags, bleached half stuff. - & 90.3 & 9.4 & .3 & (n-n. & .4 & .2 & \\
\hline Twos and blues, bleached half stuff & 91.1 & 8. 6 & & & .4 & & \\
\hline
\end{tabular}

1 Based on total cellulose.

On oven-dry basis.

3 Produced commercially by special cooking and bleaching treatment to obtain improved quality.

\section{FILLERS}

The chemical composition of the fillers used is shown in table 2 .

TABLE 2.-Composition of fillers ued ${ }^{1}$

\begin{tabular}{|c|c|c|c|c|c|c|c|}
\hline Test & Clay & $\begin{array}{l}\text { Titani- } \\
\text { um diox- } \\
\text { ide pig- } \\
\text { ment } \\
A\end{array}$ & $\begin{array}{l}\text { Titani- } \\
\text { um diox- } \\
\text { ide pig- } \\
\text { ment } \\
B\end{array}$ & $\begin{array}{c}\text { Zine } \\
\text { sulfide } \\
\text { pigment } \\
A\end{array}$ & $\begin{array}{c}\text { Zinc } \\
\text { sulfide } \\
\text { pigment } \\
\underset{B}{ }\end{array}$ & $\begin{array}{l}\text { Precipi- } \\
\text { tated } \\
\text { calcium } \\
\text { carbon- } \\
\text { ate }\end{array}$ & $\begin{array}{c}\text { Water- } \\
\text { ground } \\
\text { natural } \\
\text { calcium } \\
\text { carbonate }\end{array}$ \\
\hline $\begin{array}{l}\text { Loss at } 105^{\circ} \mathrm{C} \\
\text { Further loss on ignition }\end{array}$ & $\begin{array}{l}\% \\
0.3 \\
13.7\end{array}$ & $\begin{array}{l}\% \\
\text { None } \\
0.15\end{array}$ & $\begin{array}{c}\% \\
0.09 \\
\text { None }\end{array}$ & $\begin{array}{c}\% \\
0.09 \\
16.1\end{array}$ & $\begin{array}{l}\% \\
0.07 \\
9.0\end{array}$ & $\begin{array}{l}\% \\
0.15 \\
43.6\end{array}$ & $\begin{array}{c}\%_{0.03} \\
43.8\end{array}$ \\
\hline Silica $\left(\mathrm{SiO}_{2}\right)_{\mathrm{S}}$ & 45.3 & 0.15 & None & & & & \\
\hline $\begin{array}{l}\text { Iron oxide }\left(\mathrm{Fe}_{2} \mathrm{O}_{3}\right) \\
\text { Alumina }\left(\mathrm{Al}_{2} \mathrm{O}_{3}\right)\end{array}$ & 0.2 & $\cdots$ & $\ldots$ & & & & (n)..... \\
\hline $\begin{array}{l}\text { Alumina }\left(\mathrm{A}_{2} \mathrm{O}_{3}\right) \\
\text { Titanium }\left(\mathrm{TiO}_{2}\right)_{\ldots}\end{array}$ & $\begin{array}{r}38.8 \\
1.8\end{array}$ & $98.2^{-1}$ & 30.4 & & & & ( n \\
\hline Calcium carbonate $\left(\mathrm{CaCO}_{3}\right)_{-}$ & & & & & & 97.6 & 99.8 \\
\hline $\begin{array}{l}\text { Barium sulfate }\left(\mathrm{BaSO}_{4}\right) \\
\text { Zinc sulfide }(\mathrm{ZnS})\end{array}$ & ........... & -........... & 69.5 & 99.8 & $\begin{array}{l}44.9 \\
54.8\end{array}$ & -.......... & ....... \\
\hline
\end{tabular}

1 Analyses by Chemistry Division, National Bureau of Standards.

\section{DATA ON PAPERS MADE}

\section{PHYSICAL AND CHEMICAL MEASUREMENTS}

Data relative to the composition of the beater furnishes (materials blended in the beater) and the various physical and chemical measurements on the papers made are given in tables 3 and 4 .

The percentage of filler in paper is sometimes determined from the ash content, and sometimes, when possible, by chemical analysis. In the case of clay it was determined from the ash of the paper, corrected for the loss of water of composition from the clay during ignition. The values for the pigments were obtained by chemical analysis. 
The amount of retention of filler is that proportion of the filler added to the beater furnish which appears in the finished paper. The different methods used in different laboratories for computing retention account in some degree for the varying results reported by them. The formula used in this work was developed by Edwin Sutermeister of the S. D. Warren Co., Cumberland Mills, Maine, and has been used in previous studies [8] carried on at the Bureau, in which it was found to check the determinations by weight. The formula is:

$$
\text { Retention }=\frac{0.94(100-C-A)}{A(100-C-B)},
$$

in which $A$ is the percentage of ash in bone-dry stock going to machine (that is, the stuff box stock); $B$ is the percentage of ash in bone-dry paper at reel; and $C$ is the percentage of bone-dry filler lost on ignition.

Before being adopted for general use in a mill, however, this or any retention formula should be tested to determine whether it is suited to the particular conditions with which that mill has to deal. Many factors other than filler influence retention, but it is impossible to estimate their effects except in a general way. Some of the conditions which affect the retention of fillers are the kind of stock used, the extent of its beating (hydration), consistency of pulp and the amount of filler added, acidity, weight of paper made, speed of machine, chemicals used (such as starch, sodium silicate, or viscose materials), the use of save-alls, etc.

\section{(a) CLAY-FILLED PAPERS}

(1) Sulfite pulp, 50 percent; soda pulp, 50 percent.-Two papermachine runs were made of sulfite and soda pulps without filler-one (run 1133), without rosin size or alum; the other (run 1143), with rosin size and alum added. The test data on the runs are given in tables 3 and 4 . For the paper made from pulp alone (run 1133) the chemical test data for the heat-treated paper differ little from those for the original sheet, but when rosin and alum were added the alphacellulose content decreased and the copper number increased for the aged or heat-treated paper. The stability as regards retention of folding endurance and tearing strength is not high for either of the papers, with or without rosin size and alum. 
TABLE 3.-Papermaking details and physical test data on the book papers

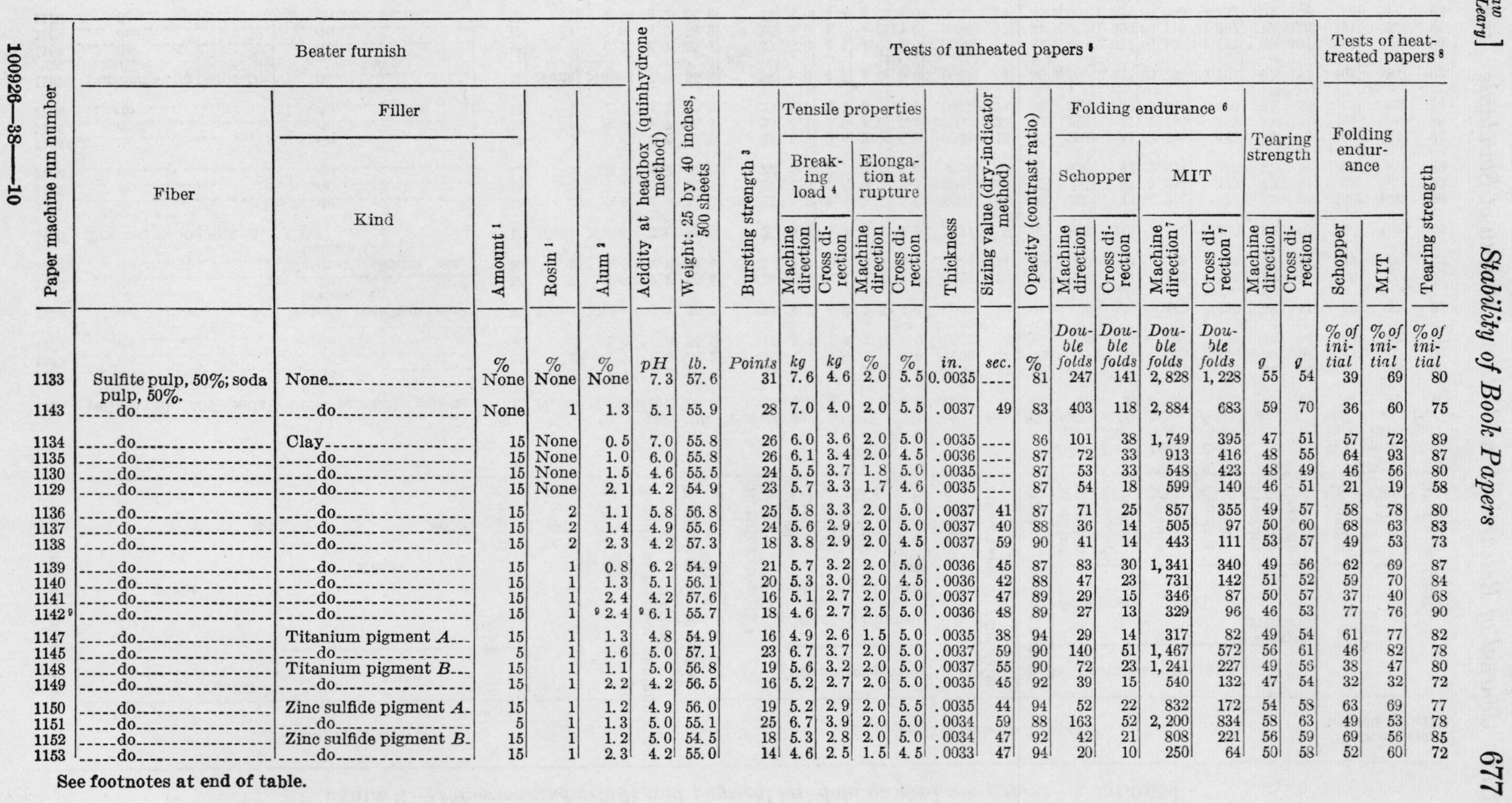


TABLE 3.-Papermaking details and physical test data on the book papers-Continued

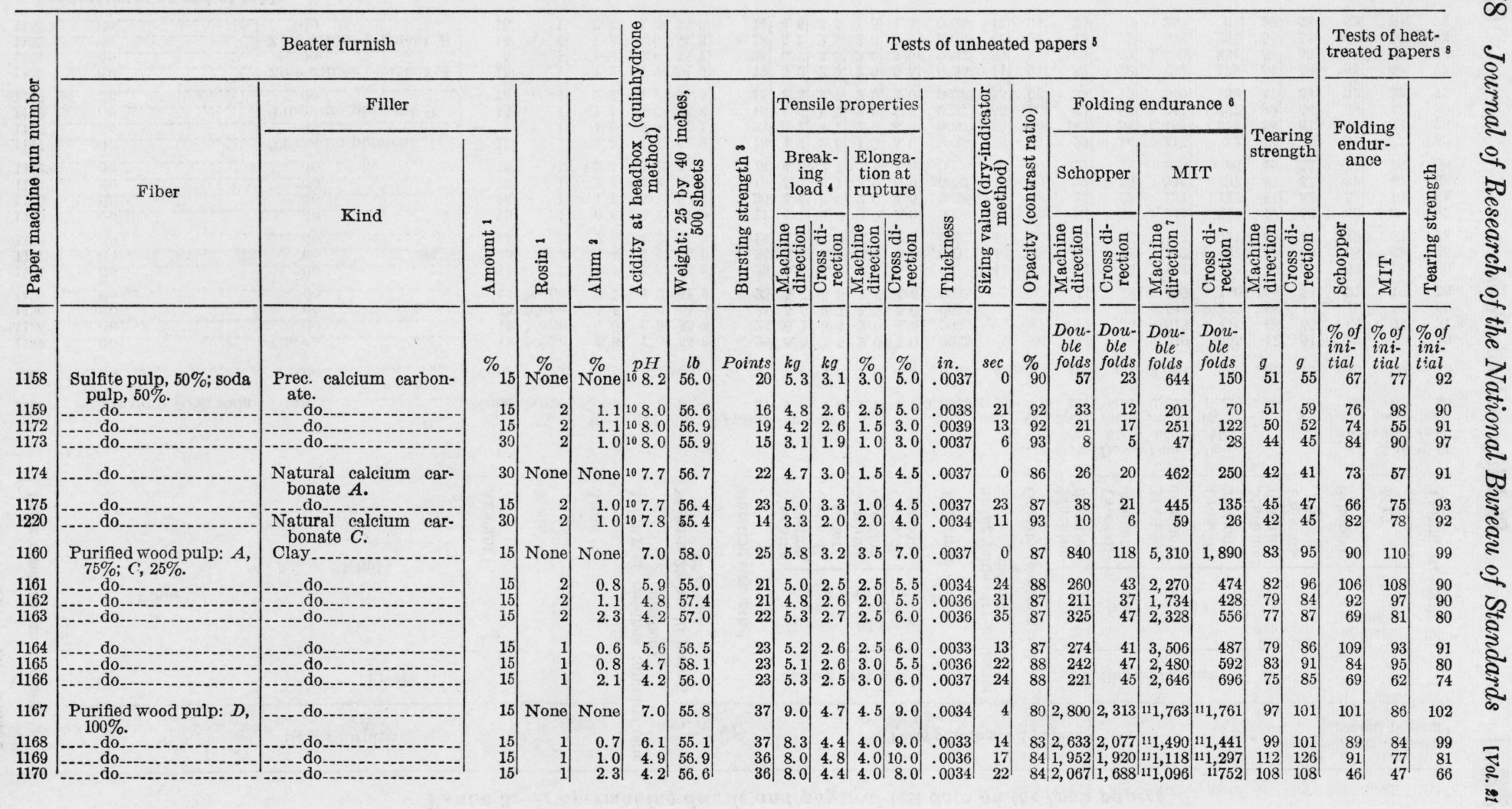




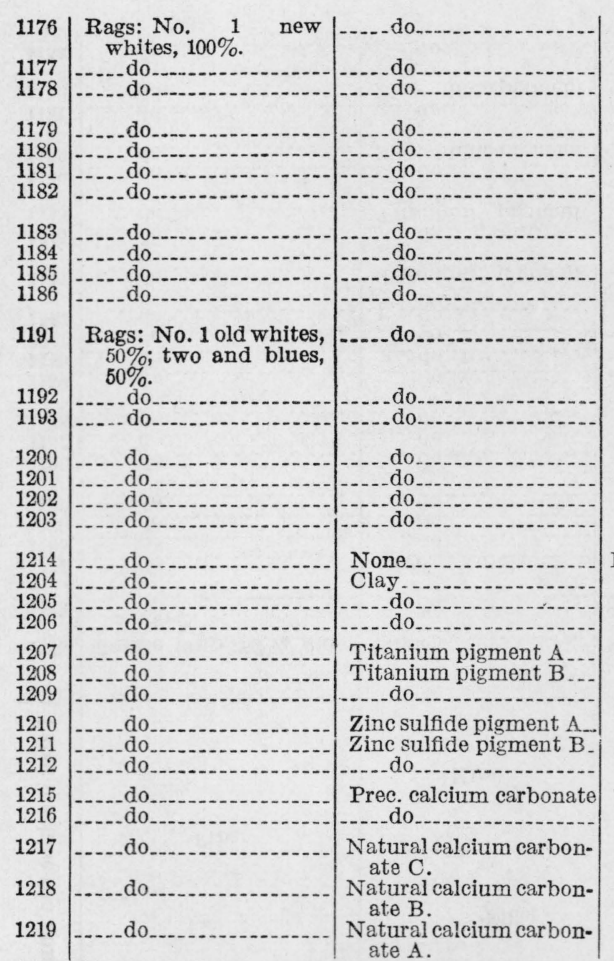

\begin{tabular}{r|r|r|r|r|}
15 & None & 0.5 & 6.6 & 56.4 \\
15 & None & 0.8 & 4.9 & 55.9 \\
15 & None & 1.5 & 4.2 & 55.0 \\
15 & 2 & 0.9 & 6.6 & 55.7 \\
15 & 2 & 1.2 & 4.9 & 56.1 \\
15 & 2 & 2.0 & 4.2 & 54.9 \\
15 & 2 & 4.0 & 4.0 & 54.8 \\
15 & 1 & 0.7 & 6.5 & 55.5 \\
15 & 1 & 1.1 & 4.8 & 55.0 \\
15 & 1 & 2.0 & 4.2 & 56.0 \\
15 & 1 & 4.0 & 4.0 & 54.3 \\
15 & None & 0.7 & 6.7 & 56.4 \\
\hline & & & & \\
15 & None & 1.3 & 4.9 & 57.1 \\
15 & None & 2.1 & 4.2 & 56.0 \\
15 & 2 & 1.0 & 6.5 & 57.2 \\
15 & 2 & 1.5 & 4.8 & 56.5 \\
15 & 2 & 2.1 & 4.2 & 54.8 \\
15 & 2 & 4.0 & 4.0 & 56.1 \\
None & 1 & 1.1 & 5.0 & 55.1 \\
15 & 1 & 1.0 & 6.3 & 56.9 \\
15 & 1 & 1.3 & 4.9 & 55.9 \\
15 & 1 & 2.2 & 4.2 & 56.9 \\
5 & 1 & 1.4 & 4.9 & 57.4 \\
15 & 1 & 1.3 & 4.9 & 56.0 \\
15 & 1 & 2.2 & 4.2 & 57.1 \\
5 & 1 & 1.6 & 4.9 & 56.4 \\
15 & 1 & 1.5 & 4.9 & 57.5 \\
15 & 1 & 2.3 & 4.2 & 57.4 \\
15 & None & None & 108.1 & 55.2 \\
30 & None & None & 108.4 & 55.3 \\
15 & None & None & 108.1 & 56.4 \\
15 & None & None & 108.1 & 55.6 \\
15 & None & None & 108.0 & 55.9 \\
\hline & & & &
\end{tabular}

\begin{tabular}{l|l|l|l|l|l|l|}
21 & 4.4 & 2.8 & 2.5 & 5.0 & .0036 \\
& & &
\end{tabular}

$\begin{array}{lllllll}21 & 4.4 & 2.7 & 2.0 & 5.0 & .0035\end{array}$

$\begin{array}{lllllll}20 & 4.4 & 2.6 & 2.0 & 5.0 & .003\end{array}$

$\begin{array}{llllllll}20 & 4.4 & 2.7 & 2.0 & 4.5 & .0034\end{array}$

$\begin{array}{llllllll}20 & 4.4 & 2.5 & 2.0 & 5.0 & .0034\end{array}$

$\begin{array}{lllllll}16 & 4.1 & 2.6 & 2.0 & 5.0 & .0033 \\ 17 & 4.2 & 2.7 & 2.0 & 5.0 & .0033\end{array}$

$\begin{array}{lllllll}19 & 4.3 & 2.8 & 2.0 & 5.5 & .0033\end{array}$

18 (17.2 4.7 2.0 5.5 .003

\begin{tabular}{lllll|l|l|}
17 & 4.1 & 2.8 & 2.0 & 5.5 & .0035 \\
19 & 4.5 & 2.9 & 2.0 & 6.0 & .0034
\end{tabular}

$\begin{array}{lllllll}15 & 3.3 & 2.2 & 2.5 & 4.0 & .0036\end{array}$

$\begin{array}{lllllll}14 & 3.2 & 2.2 & 2.0 & 4.0 & .0038\end{array}$

\begin{tabular}{ll|l|l|l|l|}
15 & 3.2 & 2.2 & 2.0 & 4.0 & .0038 \\
3.4 & 2.2 & 2.5 & 4.5 & .0036
\end{tabular}

$\begin{array}{lllllll}16 & 3.6 & 2.2 & 2.5 & 4.0 & .0037\end{array}$

$\begin{array}{llllll}14 & 3.1 & 2.2 & 2.5 & 4.5 & .0035 \\ 14 & 3.2 & 2.1 & 2.5 & 4.5 & 0037\end{array}$

\begin{tabular}{lll|l|l|l|l}
14 & 3.2 & 2.1 & 2.5 & 4.5 & .0037 \\
& 3.0 & 2.2 & 2.5 & 4.5 & .0037
\end{tabular}

$\begin{array}{lllllll}22 & 4.1 & 3.0 & 2.5 & 5.0 & .0038\end{array}$

17 3.2 2.3 2.5 4.5 .0039

\begin{tabular}{l|l|l|l|l|l}
15 & 3.1 & 2.2 & 2.5 & 4.5 & .0038 \\
16 & 2.9 & 2.3 & 2.5 & 4.5 & .0039 \\
19 & 3.7 & 2.7 & 2.5 & 5.0 & .0038
\end{tabular}

\begin{tabular}{l|lll|l|l}
19 & 3.7 & 2.7 & 2.5 & 5.0 & .0038
\end{tabular}

\begin{tabular}{l|l|l|l|l|l}
15 & 3.1 & 2.2 & 2.5 & 5.0 & .0035 \\
15 & 3.1 & 2.3 & 2.5 & 5.0 & .0037
\end{tabular}

\begin{tabular}{l|llllll}
17 & 3.3 & 2.5 & 2.5 & 5.0 & .0039
\end{tabular}

\begin{tabular}{l|l|l|l|l|l|}
15 & 3.0 & 2.1 & 2.5 & 4.5 & .0037 \\
14 & 3.0 & 2.1 & 2.5 & 4.5 & .0037
\end{tabular}

\begin{tabular}{l|llllll}
15 & 3.3 & 2.2 & 3.0 & 4.5 & .0036
\end{tabular}

\begin{tabular}{lllllll}
11 & 2.2 & 1.7 & 2.0 & 4.0 & .0033 \\
\hline
\end{tabular}

$\begin{array}{lllllll}17 & 3.7 & 2.5 & 2.5 & 4.5 & .0037\end{array}$

$\begin{array}{llllllll}17 & 3.6 & 2.5 & 3.0 & 4.5 & .0037\end{array}$

\begin{tabular}{l|l|l|l|l|l|}
18 & 3.5 & 2.5 & 2.5 & 5.0 & .0037
\end{tabular}

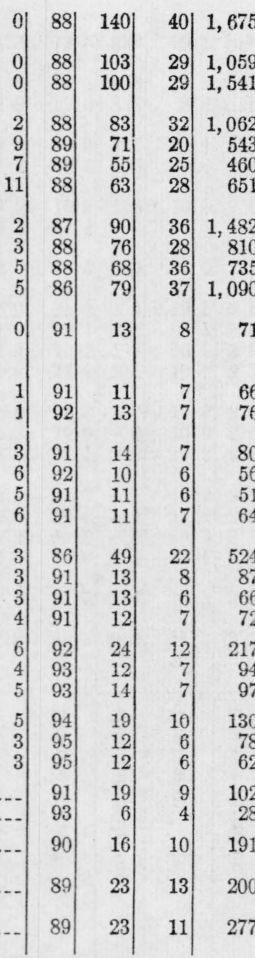

\begin{tabular}{l|l|l|l|l|l|}
642 & 94 & 110 & 108 & 92 & 100
\end{tabular}

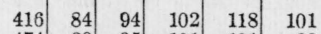
$351 \quad 85 \quad 90 \quad 100 \quad 113 \quad 98$ \begin{tabular}{l|l|l|l|l|l}
220 & 85 & 90 & 129 & 109 & 94
\end{tabular} \begin{tabular}{l|l|l|l|l|l}
253 & 80 & 85 & 96 & 99 & 87 \\
291 & 82 & 81 & 96 & 86 & 89
\end{tabular} \begin{tabular}{l|l|l|l|l|l}
403 & 88 & 85 & 106 & 92 & 95
\end{tabular} \begin{tabular}{r|r|r|r|r|r}
240 & 81 & 90 & 99 & 108 & 96
\end{tabular}

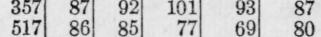
$\begin{array}{llllll}55 & 55 & 60 & 107 & 114 & 97\end{array}$ \begin{tabular}{r|r|r|r|r|r}
48 & 57 & 61 & 100 & 114 & 92 \\
53 & 56 & 59 & 78 & 95 & 90
\end{tabular} \begin{tabular}{rr|rr|r|r}
61 & 58 & 63 & 107 & 130 & 93 \\
38 & 54 & 58 & 105 & 98 & 93
\end{tabular} \begin{tabular}{r|r|r|r|r|r}
33 & 52 & 56 & 105 & 98 & 93 \\
37 & 54 & 57 & 84 & 100 & 92
\end{tabular} (2) 136 \begin{tabular}{r|r|r|r|r|r}
267 & 62 & 67 & 96 & 138 & 93 \\
61 & 56 & 55 & 92 & 86 & 95
\end{tabular} \begin{tabular}{l|l|l|l|l|l}
42 & 53 & 57 & 89 & 109 & 91 \\
47 & 55 & 57 & 87 & 102 & 86
\end{tabular} \begin{tabular}{r|r|r|r|r|r}
47 & 55 & 57 & 87 & 102 & 86
\end{tabular}

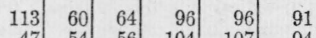
\begin{tabular}{r|r|r|r|r|r}
47 & 54 & 56 & 104 & 107 & 94 \\
53 & 55 & 57 & 103 & 97 & 93
\end{tabular} \begin{tabular}{r|r|r|r|r|r}
88 & 60 & 64 & 95 & 108 & 90
\end{tabular} \begin{tabular}{r|r|r|r|r|r}
47 & 56 & 58 & 108 & 112 & 91 \\
39 & 57 & 56 & 96 & 114 & 85
\end{tabular} \begin{tabular}{l|l|l|r|r|r}
65 & 54 & 57 & 100 & 125 & 98
\end{tabular} \begin{tabular}{l|l|l|l|l|l}
95 & 58 & 62 & 120 & 85 & 95
\end{tabular} $\begin{array}{lllllll}136 & 57 & 54 & 102 & 96 & 99\end{array}$ \begin{tabular}{l|l|l|l|l|l}
106 & 53 & 59 & 113 & 88 & 98
\end{tabular}

7 Tests made at $0.5-\mathrm{kg}$ tension unless noted.

1 Based on dry weight of fiber and filler.
2 Based on dry weight of fiber, filler, and rosin 8 Samples heated at $100^{\circ} \mathrm{C}$ for 72 hours.
See text,p. 683 . An excessive amount of alum was added in the beater to give 8 Bursting pressure, in pounds per square inch, through a circular orifice 1.2 inches in diameter. o All physical tests made at 65-percent relative humidity and $70^{\circ} \mathrm{F}$. 11 Tests made at 1.0-kg tension. 
TABLE 4.--Papermaking details and chemical test data on the book papers

\begin{tabular}{|c|c|c|c|c|c|c|c|c|c|c|c|c|c|c|c|c|c|c|c|c|c|}
\hline \multirow{3}{*}{ 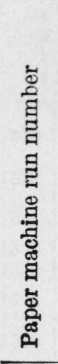 } & \multicolumn{5}{|c|}{ Beater furnish } & \multirow{3}{*}{ 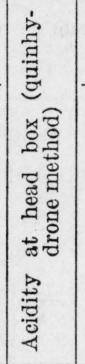 } & \multicolumn{10}{|c|}{ Original papers } & \multicolumn{5}{|c|}{$\begin{array}{l}\text { Heat-treated papers }{ }^{6} \text { (change } \\
\text { in content) }\end{array}$} \\
\hline & \multirow{2}{*}{ Fiber } & \multicolumn{2}{|l|}{ Filler } & \multirow[b]{2}{*}{ 章 } & \multirow[b]{2}{*}{ 紊 } & & \multirow[b]{2}{*}{$\frac{5}{4}$} & \multirow[b]{2}{*}{ 离 } & \multirow{2}{*}{ 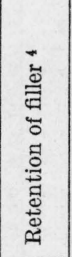 } & \multicolumn{2}{|r|}{$\begin{array}{l}\text { Acidity } \\
\text { (glass- } \\
\text { electrode } \\
\text { method) }\end{array}$} & \multirow[b]{2}{*}{ 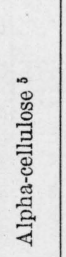 } & \multirow{2}{*}{ 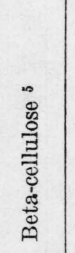 } & \multirow{2}{*}{ 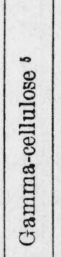 } & \multirow{2}{*}{ 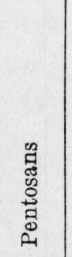 } & \multirow{2}{*}{ 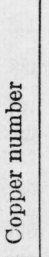 } & \multirow{2}{*}{ 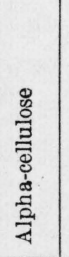 } & \multirow{2}{*}{ 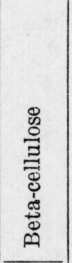 } & \multirow{2}{*}{ 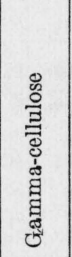 } & \multirow[b]{2}{*}{ 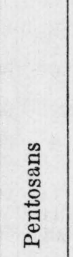 } & \multirow{2}{*}{ 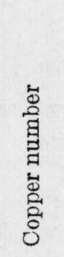 } \\
\hline & & Kind & 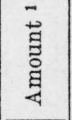 & & & & & & & 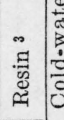 & 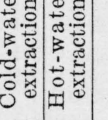 & & & & & & & & & & \\
\hline 1133 & $\begin{array}{l}\text { Sulfite pulp, } 50 \% \text {; soda } \\
\text { pulp, } 50 \%\end{array}$ & $\begin{array}{l}\text { None } \\
\text {.... do }\end{array}$ & $\begin{array}{c}\% \\
\text { None } \\
\text { None }\end{array}$ & $\begin{array}{r}\% \\
\text { None } \\
1\end{array}$ & $\begin{array}{c}\% \\
\text { None } \\
1.3\end{array}$ & \begin{tabular}{|c|}
$\mathrm{pH}$ \\
7.3 \\
5.1
\end{tabular} & $\begin{array}{c}\% \\
0.3\end{array}$ & $\%$ & & \begin{tabular}{c|c}
$\%$ & 1 \\
0.3 & 1 \\
.9 &
\end{tabular} & \begin{tabular}{l|l|}
$\mathrm{pH}$ & $\mathrm{pH}$ \\
6.9 & 6.4 \\
5.8 & 5.1
\end{tabular} & $\begin{array}{l}\% \\
75.8 \\
75.4\end{array}$ & $\begin{array}{l}\% \\
17.3 \\
17.2\end{array}$ & $\begin{array}{l}\% \\
6.9 \\
7.4\end{array}$ & $\begin{array}{l}\% \\
11.2 \\
10.7\end{array}$ & $\begin{array}{l}3.2 \\
3.3\end{array}$ & $\left|\begin{array}{c}\% \\
+0.1 \\
-2.0\end{array}\right|$ & $\begin{array}{l}\% \\
-0.5 \\
+1.7\end{array}$ & $\begin{array}{c}\% \\
+0.4 \\
+.3\end{array}$ & $\begin{array}{c}\% \\
0 \\
+0.3\end{array}$ & $\begin{array}{r}-0.1 \\
+.3\end{array}$ \\
\hline $\begin{array}{l}1134 \\
1135 \\
1130 \\
1129\end{array}$ & $\begin{array}{l}\text { do } \\
\text { do } \\
\text { do }\end{array}$ & 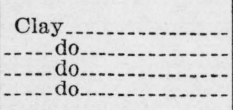 & $\begin{array}{l}15 \\
15 \\
15 \\
15\end{array}$ & $\begin{array}{l}\text { None } \\
\text { None } \\
\text { None } \\
\text { None }\end{array}$ & $\begin{array}{l}0.5 \\
1.0 \\
1.5 \\
2.1\end{array}$ & $\begin{array}{l}7.0 \\
6.0 \\
4.6 \\
4.2\end{array}$ & $\begin{array}{r}7.8 \\
8.0 \\
9.1 \\
10.1\end{array}$ & $\begin{array}{r}8.6 \\
8.9 \\
10.1 \\
11.2\end{array}$ & $\begin{array}{r}0.56 \\
.60 \\
.66 \\
.77\end{array}$ & \begin{tabular}{|l|}
.3 \\
.3 \\
.2 \\
.2
\end{tabular} & \begin{tabular}{l|l|}
6.6 & 6.2 \\
6.4 & 5.7 \\
5.3 & 4.8 \\
4.9 & 4.3
\end{tabular} & $\begin{array}{l}74.8 \\
74.4 \\
75.7 \\
76.0\end{array}$ & $\begin{array}{l}18.0 \\
17.6 \\
16.8 \\
16.3\end{array}$ & $\begin{array}{l}7.2 \\
8.0 \\
7.5 \\
7.7\end{array}$ & $\begin{array}{l}11.1 \\
10.2 \\
11.4 \\
11.4\end{array}$ & $\begin{array}{l}3.0 \\
3.2 \\
3.6 \\
3.6\end{array}$ & $\begin{array}{l}-1.0 \\
-1.2 \\
-3.3 \\
-7.6\end{array}$ & $\begin{array}{r}+.2 \\
+.7 \\
+.2 \\
+7.2 \\
+7.2\end{array}$ & $\begin{array}{r}+. \\
+.5 \\
+.5 \\
+.4\end{array}$ & $\begin{array}{r}-.1 \\
+.2 \\
+.9 \\
+.6\end{array}$ & $\begin{array}{r}-.1 \\
.0 \\
+.4 \\
+.6\end{array}$ \\
\hline $\begin{array}{l}1136 \\
1137 \\
1138\end{array}$ & 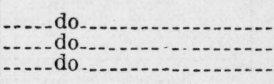 & - & $\begin{array}{l}15 \\
15 \\
15\end{array}$ & $\begin{array}{l}2 \\
2 \\
2\end{array}$ & $\begin{array}{l}1.1 \\
1.4 \\
2.3\end{array}$ & $\begin{array}{l}5.8 \\
4.9 \\
4.2\end{array}$ & $\begin{array}{r}7.8 \\
9.7 \\
10.2\end{array}$ & $\begin{array}{r}8.5 \\
10.6 \\
11.2\end{array}$ & $\begin{array}{r}.52 \\
.67 \\
.71\end{array}$ & \begin{tabular}{|l|}
1.2 \\
1.6 \\
1.9
\end{tabular} & \begin{tabular}{l|l|}
6.0 & 5.3 \\
55.5 & 4.9 \\
5.4 & 4.5
\end{tabular} & $\begin{array}{l}77.0 \\
76.8 \\
77.6\end{array}$ & $\begin{array}{l}14.7 \\
15.0 \\
13.9\end{array}$ & $\begin{array}{l}8.3 \\
8.2 \\
8.5\end{array}$ & $\begin{array}{l}10.3 \\
10.6 \\
10.8\end{array}$ & $\begin{array}{l}3.5 \\
3.6 \\
3.5\end{array}$ & $\begin{array}{l}-4.0 \\
-5.0 \\
-5.8\end{array}$ & $\begin{array}{r}+2.9 \\
+4.6 \\
+4.9\end{array}$ & $\begin{array}{r}+1.1 \\
+0.4 \\
+.9\end{array}$ & $\begin{array}{r}+.5 \\
+.2 \\
+.4\end{array}$ & $\begin{array}{l}+.7 \\
+.7 \\
+.7\end{array}$ \\
\hline $\begin{array}{l}1139 \\
1140 \\
1141 \\
1142\end{array}$ & 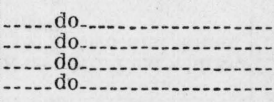 & 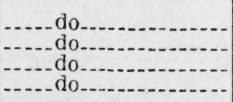 & $\begin{array}{l}15 \\
15 \\
15 \\
15\end{array}$ & $\begin{array}{l}1 \\
1 \\
1 \\
1\end{array}$ & $\begin{array}{r}0.8 \\
1.3 \\
2.4 \\
72.4\end{array}$ & $\begin{array}{r}6.2 \\
5.1 \\
4.2 \\
76.1\end{array}$ & $\begin{array}{r}8.1 \\
9.2 \\
10.8 \\
11.4\end{array}$ & $\begin{array}{r}8.8 \\
110.0 \\
11.8 \\
12.6\end{array}$ & \begin{tabular}{r|}
.53 \\
.64 \\
.73 \\
.80
\end{tabular} & \begin{tabular}{|l|}
0.9 \\
1.0 \\
1.2 \\
1.0
\end{tabular} & \begin{tabular}{l|l|}
6.2 & 5.5 \\
5.9 & 5.1 \\
5.2 & 4.6 \\
6.5 & 5.3
\end{tabular} & $\begin{array}{l}77.8 \\
76.4 \\
74.3 \\
75.0\end{array}$ & $\begin{array}{l}14.5 \\
16.5 \\
18.1 \\
17.6\end{array}$ & $\begin{array}{l}7.7 \\
7.1 \\
7.6 \\
7.4\end{array}$ & $\begin{array}{r}10.5 \\
10.5 \\
9.7 \\
10.7\end{array}$ & $\begin{array}{l}3.1 \\
3.3 \\
3.2 \\
3.2\end{array}$ & $\begin{array}{l}-2.6 \\
-3.2 \\
-6.3 \\
-2.6\end{array}$ & $\begin{array}{r}+2.3 \\
+2.5 \\
+6.8 \\
+2.5\end{array}$ & $\begin{array}{r}+.3 \\
+.7 \\
-.5 \\
+.1\end{array}$ & $\begin{array}{r}+.4 \\
-.1 \\
+1.2 \\
+0.1\end{array}$ & $\begin{array}{r}+.5 \\
+.4 \\
+.7 \\
+.7\end{array}$ \\
\hline 1147 & .....do-... & Titanium pigment & 15 & 1 & 1.3 & 4. 8 & 9.7 & 9.0 & .59 & 1.0 & $5.2 \ldots$ & 73. 6 & 18. 2 & 8.2 & 11.0 & 3.3 & -5.0 & $\begin{array}{r}+4.8 \\
\end{array}$ & $\begin{array}{r}+.2 \\
+.9\end{array}$ & +.4 & +.6 \\
\hline $\begin{array}{l}1145 \\
1148\end{array}$ & (ndo & Titanium pigment & $\begin{array}{r}5 \\
15\end{array}$ & & $\begin{array}{l}1.6 \\
1.1\end{array}$ & $\begin{array}{l}5.0 \\
5.0\end{array}$ & $\begin{array}{l}3.8 \\
7.4\end{array}$ & $\begin{array}{r}3.1 \\
82.1 ; 4.6\end{array}$ & $\begin{array}{l}.68 \\
.44\end{array}$ & $\begin{array}{r}0.9 \\
.9\end{array}$ & \begin{tabular}{l|l|}
5.6 & 4.9 \\
5.4 & 4.6
\end{tabular} & $\begin{array}{r}75.2 \\
73.5\end{array}$ & $\begin{array}{l}15.8 \\
19.3\end{array}$ & $\begin{array}{l}9.0 \\
7.2\end{array}$. & 10.6 & $\begin{array}{l}3.0 \\
3.0\end{array}$ & $\begin{array}{l}-5.2 \\
-6.0\end{array}$ & $\begin{array}{l}+5.8 \\
+5.6\end{array}$ & $\begin{array}{l}-.6 \\
+.4\end{array}$ & 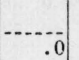 & $\begin{array}{l}+.8 \\
+.3\end{array}$ \\
\hline $\begin{array}{l}1149 \\
1150\end{array}$ & (ndo-... & Zinc sulfide pigment & $\begin{array}{l}15 \\
15\end{array}$ & $\begin{array}{l}1 \\
1\end{array}$ & $\begin{array}{l}2.2 \\
1.2\end{array}$ & $\begin{array}{l}4.2 \\
4.9\end{array}$ & $\begin{aligned} 10.5 \\
8.5\end{aligned}$ & $\begin{array}{r}83.2 ; 6.6 \\
9.4\end{array}$ & .64 & $\begin{array}{l}1.1 \\
1.1\end{array}$ & \begin{tabular}{l|l|}
5.2 & 4.6 \\
5.8 & ---
\end{tabular} & $\begin{array}{l}72.2 \\
78.2\end{array}$ & $\begin{array}{l}20.6 \\
13.6\end{array}$ & \begin{tabular}{|l|}
7.2 \\
8.2
\end{tabular} & $\begin{array}{l}10.6 \\
10.4\end{array}$ & $\begin{array}{l}3.0 \\
(10)\end{array}$ & $\begin{array}{l}-8.2 \\
-3.7\end{array}$ & $\begin{array}{r}+7.3 \\
+2.9\end{array}$ & $\begin{array}{r}+.9 \\
+.8\end{array}$ & $\begin{array}{l}.0 \\
.0\end{array}$ & ${ }_{(10)}^{+1.1}$ \\
\hline $\begin{array}{l}1151 \\
1152\end{array}$ & (do do & Zine sulfide pigment & \begin{tabular}{r|r}
5 \\
15
\end{tabular} & $\begin{array}{l}1 \\
1\end{array}$ & $\begin{array}{l}1.3 \\
1.2\end{array}$ & $\begin{array}{l}5.0 \\
5.0\end{array}$ & $\begin{array}{l}2.5 \\
8.6\end{array}$ & $\begin{array}{r}2.3 \\
05.0 ; 4.0\end{array}$ & $\begin{array}{l}.52 \\
.55\end{array}$ & \begin{tabular}{|l|}
0.9 \\
1.1
\end{tabular} & \begin{tabular}{ll|l|}
5.7 & \\
5.8 & 5.1
\end{tabular} & $\begin{array}{r}76.8 \\
77.4\end{array}$ & $\begin{array}{l}15.0 \\
15.7\end{array}$ & \begin{tabular}{|}
8.2 \\
6.9
\end{tabular} & $\begin{array}{r}9.6 \\
10.3\end{array}$ & $\begin{array}{l}(10) \\
(10)\end{array}$ & $\begin{array}{l}-2.0 \\
-3.7\end{array}$ & $\begin{array}{r}+1.2 \\
+3.5\end{array}$ & $\begin{array}{r}+.8 \\
+.2\end{array}$ & $\begin{array}{l}-.7 \\
+.6\end{array}$ & $\begin{array}{l}(10) \\
(10)\end{array}$ \\
\hline 1153 & ...do. & & 15 & 1 & 2.3 & 4. 2 & 11.2 & • $6.2 ; 5.1$ & .74 & $|1.2|$ & \begin{tabular}{l|l|l}
5.1 & 4.6
\end{tabular} & 77.3 & 13.9 & 8.8 & 10.7 & (10) & -1.3 & +0.1 & $\mid+1.2$ & $|+1|$ & (10) \\
\hline
\end{tabular}




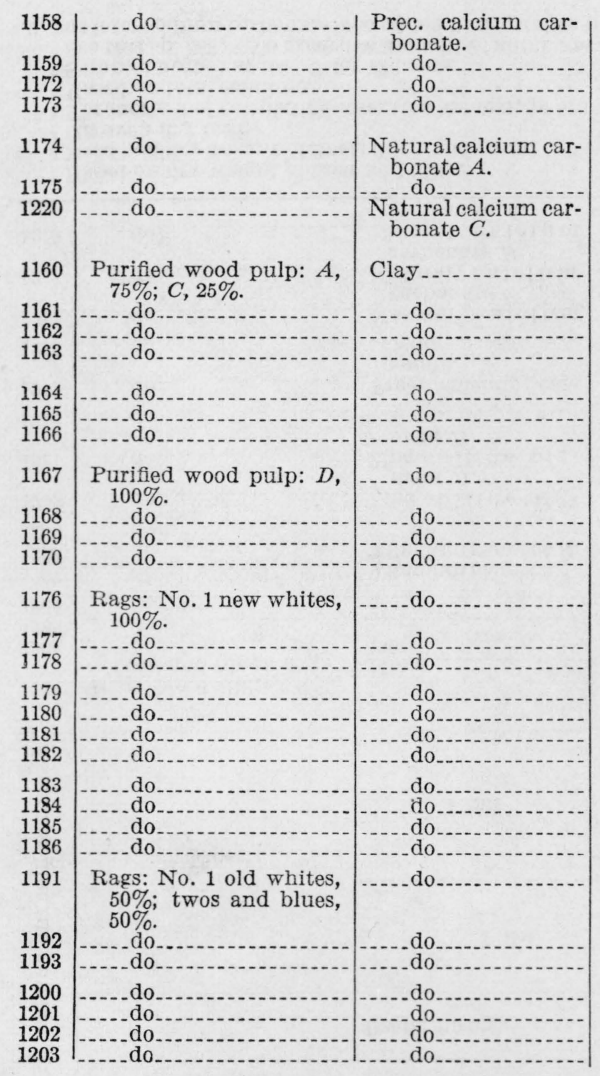

See footnotes at end of table.
15|None None ${ }^{11} 8.2|4.2|$

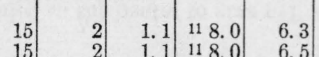
\begin{tabular}{r|r|r|r|r|}
30 & 2 & 1.0 & 118.0 & 12.1 \\
\hline
\end{tabular} 30 None None $117.7 \quad 10.0$ \begin{tabular}{l|l|l|l|l}
15 & 2 & 1.0 & 117.7 & 6.0
\end{tabular} \begin{tabular}{l|l|l|l|l|}
30 & 2 & 1.0 & 117.8 & 12.6
\end{tabular} 15 None None $7.0 \quad 7.6$ \begin{tabular}{l|l|l|l|l|}
15 & 2 & 0.8 & 5.9 & 10.5 \\
15 & 2 & 1.1 & 4.8 & 10.7
\end{tabular} \begin{tabular}{l|l|l|l|l|}
15 & 2 & 1.1 & 4.8 & 10.7 \\
15 & 2 & 2.3 & 4.2 & 10.0
\end{tabular} \begin{tabular}{ll|l|l|l}
15 & 1 & 0.6 & 5.6 & 10.9
\end{tabular} \begin{tabular}{l|l|l|l|l|}
15 & 1 & .8 & 4.7 & 10.7 \\
15 & 1 & 2.1 & 4.2 & 10.1
\end{tabular} 15 None None \begin{tabular}{r|r|r|r|r|}
15 & 1 & 0.7 & 6.1 & 12.1
\end{tabular} \begin{tabular}{ll|l|l|l|}
15 & 1 & 1.0 & 4.9 & 12.5 \\
15 & 1 & 2.3 & 4.2 & 12.3
\end{tabular}

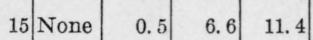

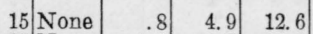
15 None $1.5 \quad 4.2 \quad 11.6$ \begin{tabular}{l|l|l|l|l|}
15 & 2 & 0.9 & 6.6 & 11.2 \\
15 & 2 & 1.2 & 4.9 & 12.3
\end{tabular} $\begin{array}{lllll}15 & 2 & 1.2 & 4.9 & 12.3 \\ 15 & 2 & 2.0 & 4.2 & 11.8\end{array}$

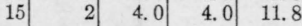
\begin{tabular}{l|l|l|l|l|l}
15 & 1 & 0.7 & 6.5 & 11.6 \\
15 & 1 & 1.1 & 4.8 & 12.3
\end{tabular} \begin{tabular}{l|l|l|l|l}
15 & 1 & 2.0 & 4.2 & 11.6
\end{tabular} $\begin{array}{lllll}15 & \text { None } & 0.7 & 6.7 & 12.5\end{array}$ 15 None $1.3 \quad 4.9 \quad 12.4$ \begin{tabular}{ll|l|l}
15 & 12.4 \\
\hline & & 0
\end{tabular} \begin{tabular}{l|l|l|l|l|}
15 & 2 & 1.0 & 6.5 & 11.7 \\
15 & 2 & 1.5 & 4.8 & 12.3
\end{tabular} \begin{tabular}{l|l|l|l|l|}
15 & 2 & 2.1 & 4.2 & 12.3 \\
15 & 2 & 4.0 & 4.0 & 12.1
\end{tabular}

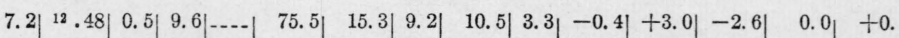
\begin{tabular}{l|llllllllllllll}
11.2 & 12.75 & 1.5 & 9.2 & $\ldots$ & 76.6 & 15.7 & 7.7 & 10.1 & 3.3 & -1.5 & +1.0 & +0.5 & -1.3 &.+
\end{tabular}

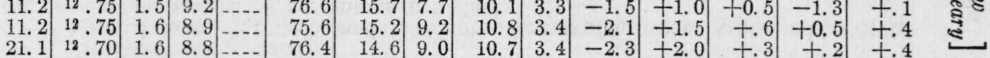
\begin{tabular}{l|l|l|l|l}
16.4 & 12.55 & 0.3 & 9.2 & $\ldots-$.
\end{tabular}

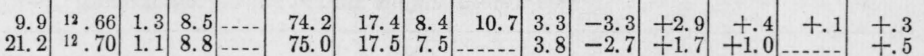

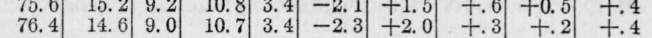
$\begin{array}{llllllllll}74.2 & 18.3 & 7.5 & 9.7 & 3.3 & -2.2 & +1.8 & +.4 & +.9 & +.3\end{array}$

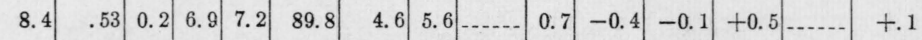

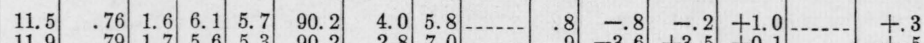

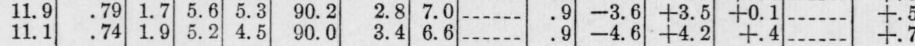
$\begin{array}{lllllllllll}12.1 & .81 & 1.1 & 6.0 & 5.7 & 89.8 & 4.8 & 5.4 & \ldots . .\end{array}$

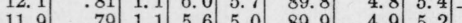
\begin{tabular}{l|l|l|l|l|l|l|l|l|l|l}
11.2 & 74 & 1.0 & 5.0 & 4.4 & 89.6 & 4.9 & 5.2 & $-\cdots-1$ \\
\hline
\end{tabular} \begin{tabular}{lll|l|l|l|l}
10.8 & .71 & 0.1 & 6.8 & 7.0 & 82.4
\end{tabular}

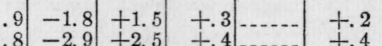
\begin{tabular}{ll|l|l|l|l}
.8 & -2.9 & +2.5 & +.4 & $\cdots$ & +.4 \\
9 & -5.0 & +5.1 & -.1 &...+ & +.5
\end{tabular}

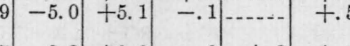
\begin{tabular}{llllllllllllll}
13.5 & .88 & .9 & 6.4 & 6.0 & 83.2 & 4.4 & 12.4 & 8.5 & .8 & -.7 & +.5 & +.2 & +.1 \\
\hline
\end{tabular}

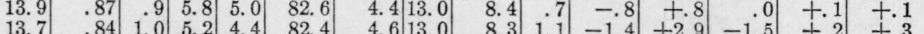
12.0 \begin{tabular}{l|l|l|l|l|l|l|l}
.81 & 0.3 & 7.2 & 7.3 & 96.0 & 3.2 & 0.8 \\
\hline
\end{tabular}

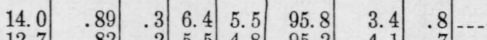
\begin{tabular}{l|l|l|l|l|l|l|l|l|l}
12.7 & .82 & .2 & 5.5 & 4.8 & 95.2 & 4.1 & .7 \\
\hline
\end{tabular} \begin{tabular}{lll|l|l|l|l|l|l|l}
12.4 & .80 & 1.6 & 6.4 & 5.9 & 96.0 & 2.6 & 1.4 & - \\
13.6 & 87 & 1.8 & 5.6 & 5.0 & 94.8 & 3.7 & 1.5 & - \\
\hline
\end{tabular} \begin{tabular}{ll|l|l|l|l|l}
13.6 & .87 & 1.8 & 5.6 & 5.0 & 94.8
\end{tabular} \begin{tabular}{l|r|r|r|r|r|}
13.0 & 83 & 1.7 & 5.4 & 4.7 & 95.1 \\
13.0 & .83 & 1.7 & 5.0 & 4.4 & 94.8 \\
\hline
\end{tabular} \begin{tabular}{l|l|l|l|l|l|l}
12.7 & .82 & 0.8 & 6.3 & 6.0 & 95.8 \\
\hline
\end{tabular} \begin{tabular}{l|l|l|l|l|l|l}
13.6 & .87 & .9 & 5.6 & 5.0 & 95.4 \\
12.7 & .84 & 9 & 5.1 & 4.5 & 95.1
\end{tabular} 3. 6 1.3 1.3

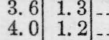
\begin{tabular}{l|l|l}
3.4 & 0.8 & - \\
3. & 1.0 & -
\end{tabular} \begin{tabular}{ll|l|l|l|l|l|l|l}
13.9 & .84 & .2 & 6.8 & $\ldots$. & 92.3 & 7.5 & .2 & $-\ldots-1$
\end{tabular} \begin{tabular}{lll|l|l|l|l|l|l}
13.8 & .83 & .2 & 5.8 & $\ldots$ & 91.1 & 8.7 & .2 & $\ldots$
\end{tabular} \begin{tabular}{ll|l|l|l|l|l|l|l|l|l}
13.8 & .83 & .3 & 5.1 &..-- & 91.6 & 8.3 & .1 \\
\hline
\end{tabular} \begin{tabular}{l|l|l|l|l|l|l|l|l|}
13.0 & .79 & 1.4 & 6.7 &.- & 90.4 & 8.2 & 1.4 & $\ldots$ \\
\hline 13 & .85 & 1.4 & 5.9 & 5.1 & 89.0 & 9.5 & 1.5 & - \\
\hline
\end{tabular} \begin{tabular}{l|l|l|l|l|l|l|l|l|l}
13.7 & .85 & 1.4 & 5.9 & 5.1 & 89.0 & 9.5 & 1.5 \\
\hline
\end{tabular} \begin{tabular}{|l|l|l|l|l|l|l|l|l|}
\hline 13.7 & .84 & 1.5 & 5.5 & 4.8 & 89.1 & 9.3 & 1.6 \\
\hline
\end{tabular}

.

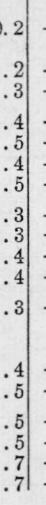

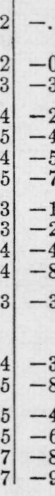




\begin{tabular}{|c|c|c|c|c|c|c|c|c|c|c|c|c|c|c|c|c|c|c|c|c|c|c|}
\hline \multirow{3}{*}{ 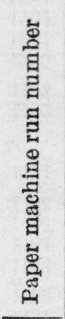 } & \multicolumn{5}{|c|}{ Beater furnish } & \multirow{3}{*}{ 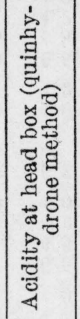 } & \multicolumn{11}{|c|}{ Original papers } & \multicolumn{5}{|c|}{$\begin{array}{l}\text { Heat-treated papers }{ }^{6} \text { (change } \\
\text { in content) }\end{array}$} \\
\hline & \multirow[b]{2}{*}{ Fiber } & \multicolumn{2}{|l|}{ Filler } & \multirow[b]{2}{*}{ मี } & \multirow[b]{2}{*}{ 紊 } & & \multirow[b]{2}{*}{ क्ष } & \multirow[b]{2}{*}{ 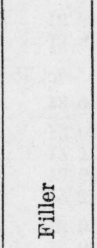 } & \multirow{2}{*}{ 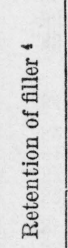 } & \multirow[b]{2}{*}{ : } & \multicolumn{2}{|c|}{$\mid \begin{array}{c}\text { Acidity } \\
\text { (glass- } \\
\text { electrode } \\
\text { method) }\end{array}$} & \multirow{2}{*}{ 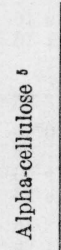 } & \multirow[b]{2}{*}{ 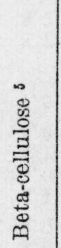 } & \multirow{2}{*}{ 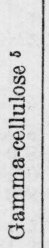 } & \multirow[b]{2}{*}{ 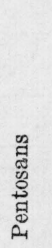 } & \multirow{2}{*}{ 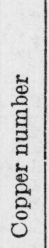 } & \multirow[b]{2}{*}{ 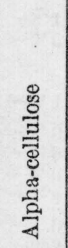 } & \multirow[b]{2}{*}{ 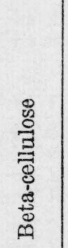 } & \multirow{2}{*}{ 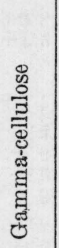 } & \multirow[b]{2}{*}{ 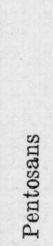 } & \multirow[b]{2}{*}{ 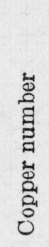 } \\
\hline & & Kind & $\begin{array}{l}\overrightarrow{\vec{a}} \\
\text { 苜 }\end{array}$ & & & & & & & & 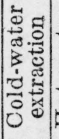 & 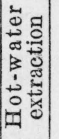 & & & & & & & & & & \\
\hline 1214 & Rags: No. 1 whites, $50 \%$; & & $\%$ & $\%$ & $\%$ & $\mathrm{pH}$ & $\%$ & $\%$ & & $\%$ & $\mathrm{pH}$ & $\mathrm{pH}$ & $\%$ & $\%$ & $\%$ & & & $\%$ & $\%$ & $\%$ & & \\
\hline $\begin{array}{l}1204 \\
1205 \\
1206\end{array}$ & \begin{tabular}{|}
$\begin{array}{r}\text { twos and blues } \\
\text { do-a }\end{array}$ \\
do- \\
\end{tabular} & $\begin{array}{l}\text { None-1 } \\
\text { Clay }\end{array}$ & $\begin{array}{r}\text { None } \\
15 \\
15 \\
15\end{array}$ & $\begin{array}{l}1 \\
1 \\
1 \\
1\end{array}$ & $\begin{array}{l}1.1 \\
1.0 \\
1.3 \\
2.2\end{array}$ & $\begin{array}{l}5.0 \\
6.3 \\
4.9 \\
4.2\end{array}$ & $\begin{array}{r}0.4 \\
12.1 \\
12.6 \\
12.2\end{array}$ & $\begin{array}{r}13.4 \\
14.0 \\
13.5\end{array}$ & $\begin{array}{l}.82 \\
.87 \\
.84\end{array}$ & $\begin{array}{r}0.5 \\
.5 \\
.5 \\
.7\end{array}$ & $\mid \begin{array}{r}6.2 \\
6.2 \\
5.4 \\
5.0\end{array}$ & & $\begin{array}{l}90.3 \\
90.4 \\
89.6 \\
90.0\end{array}$ & $\begin{array}{l}8.6 \\
8.6 \\
9.5 \\
9.1\end{array}$ & $\begin{array}{l}1.1 \\
1.0 \\
0.9 \\
.1\end{array}$ & & $\begin{array}{l}.4 \\
.4 \\
.6 \\
.6\end{array}$ & $\begin{array}{l}-3.0 \\
-2.9 \\
-4.2 \\
-6.7\end{array}$ & $\begin{array}{l}+2.8 \\
+2.8 \\
+3.6 \\
+6.4\end{array}$ & $\begin{array}{l}+.2 \\
+.1 \\
+.6 \\
+.3\end{array}$ & & \\
\hline $\begin{array}{l}1207 \\
1208 \\
1209\end{array}$ & \begin{tabular}{|c|} 
do do \\
- do
\end{tabular} & $\begin{array}{l}\text { Titanium pigment } A \text {. } \\
\text { Titanium pigment } B \text {. }\end{array}$ & $\begin{array}{r}5 \\
15 \\
15\end{array}$ & $\begin{array}{l}1 \\
1 \\
1\end{array}$ & $\begin{array}{l}1.4 \\
1.3 \\
2.2\end{array}$ & $\begin{array}{l}4.9 \\
4.9 \\
4.2\end{array}$ & $\begin{array}{r}5.0 \\
13.4 \\
13.7\end{array}$ & $\begin{array}{r}4.6 \\
84.2 ; 8.1 \\
84.2 ; 8.4\end{array}$ & $\begin{array}{r}.87 \\
.82 \\
.83\end{array}$ & $\begin{array}{l}.8 \\
.9 \\
.8\end{array}$ & $\begin{array}{l}5.2 \\
5.7 \\
5.6\end{array}$ & & $\begin{array}{l}90.2 \\
90.5 \\
89.8\end{array}$ & $\begin{array}{r}8.8 \\
9.5 \\
10.1\end{array}$ & $\begin{array}{r}1.0 \\
0.0 \\
.1\end{array}$ & & $\begin{array}{l}.5 \\
.4 \\
.6\end{array}$ & $\begin{array}{l}-4.4 \\
-3.7 \\
-4.3\end{array}$ & $\begin{array}{r}+3.9 \\
+3.6 \\
+4.4\end{array}$ & $\begin{array}{l}+.5 \\
+.1 \\
+.1\end{array}$ & & \\
\hline 1210 & ....... do & $\begin{array}{l}\text { Zinc sulfide pig- } \\
\text { ment } A \text {. }\end{array}$ & 5 & 1 & 1.6 & 4.9 & 4.2 & 4.3 & .85 & .8 & 6.1 & & 90.5 & 9.0 & .5 & & (10) & -5.1 & +4.4 & +.7 & & (10) \\
\hline 1211 & ......do_... & $\begin{array}{l}\text { Zine sulfide pig- } \\
\text { ment } B \text {. }\end{array}$ & 15 & 1 & 1.5 & 4.9 & 12.8 & $97.2 ; 5.8$ & .85 & .9 & 6.2 & & 91.0 & 8.1 & .9 & & (10) & -4.7 & +4.3 & +.4 & & (10) \\
\hline 1212 & ......do_... & -... do & 15 & 1 & 2.3 & 4.2 & 12.4 & $96.9 ; 5.6$ & .83 & .9 & 5.5 & & 90.7 & 8.8 & .5 & & (10) & -6.8 & +6.1 & +.7 & & $\left({ }^{10}\right)$ \\
\hline $1215_{\mathrm{s}}^{\mathrm{z}}$ & ................. & $\begin{array}{l}\text { Prec. calcium car- } \\
\text { bonate. }\end{array}$ & 15 & None & None & 118.1 & 8.3 & 14.0 & 12.93 & .1 & 9.5 & & 92.8 & 6.7 & .5 & & .4 & -0.6 & +0.3 & +.3 & & \\
\hline 1216 & do... & do & 30 & None & None & 118.4 & 16.5 & 28.2 & ${ }^{12} .94$ & .1 & 9.5 & & 92.7 & 6.6 & .7 & & .3 & +.1 & -.3 & +.2 & & \\
\hline 1217 & -.....do do & $\begin{array}{l}\text { Natural calci um } \\
\text { carbonate } C .\end{array}$ & 15 & None & None & 118.1 & 6.9 & 11.8 & ${ }^{12} .79$ & .1 & 9.4 & $-\ldots$ & 92.5 & 6.9 & .6 & & .3 & -2.1 & +2.1 & .0 & & \\
\hline 1218 & ... do_. & Natural calci um & 15 & None & None & 118.1 & 7.3 & 12.4 & 12.82 & .2 & 9.4 & & 92.4 & 7.0 & .6 & & .4 & -2.7 & +2.5 & +.2 & & \\
\hline 1219 & do & $\begin{array}{c}\text { Natural calcium } \\
\text { carbonate } A .\end{array}$ & 15 & None & None & $\left|\begin{array}{ll}11 & 8.0\end{array}\right|$ & 7.3 & 12.2 & ${ }^{12} .81$ & .2 & & & 92.1 & 7.3 & .6 & & .4 & -1.4 & +1.5 & -.1 & & \\
\hline
\end{tabular}

1 Based on dry weight of fiber and filler.

2 Based on dry weight of fiber, filler, and rosin.

On oven-dry basis.

For cetention see text, p. 676

6 Samples heated at $100^{\circ} \mathrm{C}$ for 72 hours.

${ }^{7}$ See text, p. 683. An excessive amount of alum was added in the beater to give $\mathrm{pH}$

4.2 , but later sodium carbonate was added to neutralize part of the acidity.
8 Titanium dioxide and barium sulfate, respectively.

${ }_{10}$ Zinc sulfide and barium sulfate, respectively.
11 Prese of zinc sulfide interferes with the chemical reactions in the test.

12 A pproximated, did not have data required for use of formula. Obtained by divid. ing percentage in paper by percentage furnished. 
A series of runs $(1134,1135,1130$, and 1129) of unsized papers containing clay was made in which the amount of alum added was varied. The effect of acidity on the stability of the unsized clayfilled papers is shown by the decrease in alpha-cellulose content and increase in copper number of the heat-treated papers as the amount of alum in the furnish increased, and the decrease in retention of folding endurance and tearing strength. In general, the agreement between the $\mathrm{pH}$ values of the water from the stock at the head box and of the hot-water extraction of the finished paper was fairly good, the differences probably being due to differences in buffer conditions, the head-box sample being well buffered and the water extraction of the finished paper poorly buffered. The clay retention of the runs increased with increased amounts of alum.

To study the effect of rosin sizing on the stability of sulfite-soda papers containing clay several machine runs were made in which the amounts of alum and rosin size were varied.

The test data on the runs (1136 to 1138) in which the amount of rosin was kept constant at 2 percent and the amount of alum was varied show that the change in alpha-cellulose content of the heattreated papers increased as the alum was increased, but that the increase in copper number remained constant, although large. The percentage of retention of folding endurance and tearing strength gradually decreased as acidity increased but to less extent than for the unsized papers, to which rosin was not added (runs 1134, 1135, 1130 , and 1129). The rosin seems to have hindered deterioration. This phenomenon was noted also in a previous study [2] of sulfite pulps for writing papers. As a possible reason for this apparent disagreement, it is suggested that for pulps in the low stability range, rosin sizing may actually have a protective effect. The indications are that, within the range studied, the amount of rosin employed in sizing sulfite-soda papers is not an important consideration as far as stability is concerned. Retention of clay in runs 1136 to 1138 increased as alum was increased.

For the series of papers (runs 1139 to 1141) in which the amount of rosin was kept constant at 1 percent and the amount of alum was varied, the stability falls between that of papers with no rosin in the beater furnish and those with 2 percent of rosin added. The data on the heat-treated papers of this series are shown graphically in figure 1 .

Although the folding strength of the original clay-filled papers decreased as the amount of alum was increased, some of the decrease was due to the increased retention of clay. The increased filler content should not affect the strength retention of the heat-treated papers, however, since clay is an inert substance which has been previously found to have no harmful effect on the stability of paper.

To determine whether paper made from stock having high acidity in the beater but subsequently adjusted at the paper machine to low acidity would remain stable, a paper-machine run (1142) was made similar to run 1141 in the preparation of the stock in the beater. An excessive amount of alum was added in the beater to give a $\mathrm{pH}$ of 4.2 , but a solution of soda ash (sodium carbonate) was added to neutralize some of the acidity as the stock was being pumped from the beater chest to the machine chest. The $\mathrm{pH}$ at the head box of 
the paper machine was 6.1. The stability of the finished paper compared favorably with that of the run (1139) which had $\mathrm{pH} 6.2$ at the head box without any treatment to reduce the original acidity. The retention of folding endurance and tearing strength was as good for the paper of the adjusted run as for the run in which only a small amount of alum was used (run 1139), and the change in alphacellulose content of the heat-treated paper was no greater. But the increase in copper number was the same as that of the paper with high acidity at the head box (run 1141). The sizing value was not materially affected, but the clay retention was increased. The improvement in clay retention may be attributable to the presence of
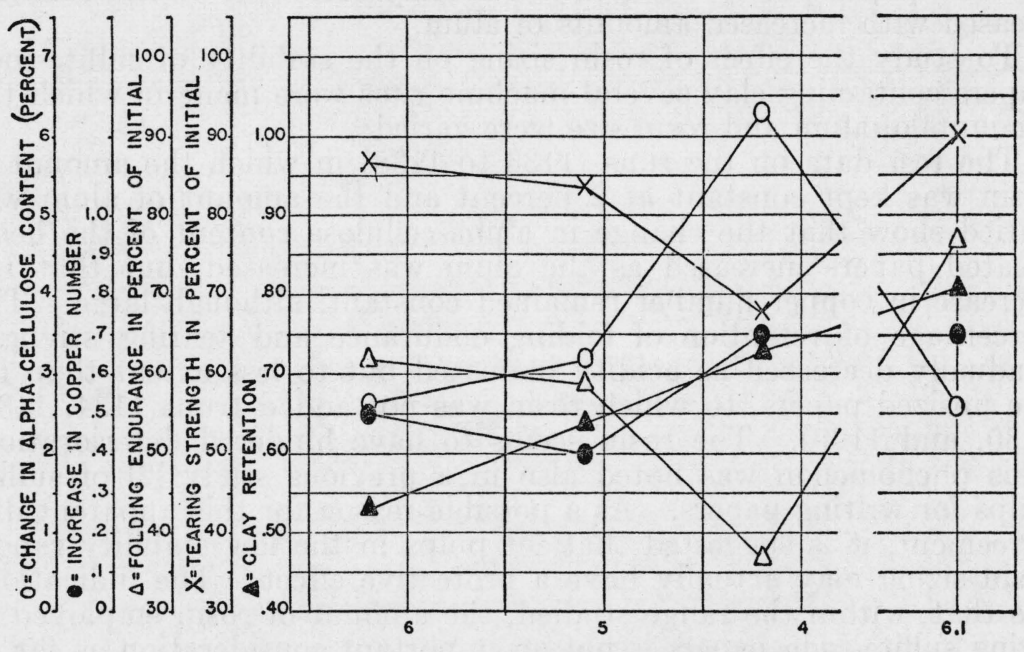

PH OF STOCK AT HEAD BOX

(RUNS NOS. 1139, 1140, 1141, ANO 1142, RESPECTIVELY)

FIGURE 1.-Effect of acidity on stability of rosin-sized, clay-filled book papers made from sulfite and soda pulps.

Points at $\mathrm{pH}$ 6.1 are displaced to right of main graph to avoid confusion. For this run there was considerable difference between the initial and final $\mathrm{pH}$ values. An excessive amount of alum was used in the beater to give a $\mathrm{pH}$ of 4.2 , but sodium carbonate was added to neutralize part of the acidity as the stock was being pumped from the beater chest to the machine chest.

aluminum floc formed when the acidity caused by the excessive amount of alum was neutralized with the soda ash. Retention has been shown by a number of writers to be partly the result of occlusion and fixation of the finer particles in the sheet by the rosin and the aluminum hydrate floc formed in the sizing operation.

In addition to the results obtained on the experimental paper, tests at the Bureau on commercial papers of known history show that with high acidity in the beater followed by treatment at the paper machine to obtain low acidity, paper of fair stability, as far as acidity is concerned, may be produced.

The pentosan content of the different sulfite-soda papers was not appreciably changed by the heat treatment. It is apparent that pentosans do not contribute to the deterioration of cellulose to the extent that modified celluloses do, and therefore that pentosan 
determinations are comparatively unimportant in evaluating the relative stability of papers.

(2) Purified wood pulp: $A, 75$ percent; $C, 25$ percent.-In previous work [4] at the Bureau on purified wood pulps, papers made from pulp $A$ without rosin and alum were relatively stable but had comparatively low folding strength. Pulp $B$ produced a hard sheet, stronger but less stable to the heat test, and not as good in color as that made from pulp $A$. The use of a small amount (25 percent of furnish) of pulp $B$ with the weaker but more stable pulp $A$ increased the strength of the sheet without appreciably lessening its stability. Paper made from pulp $C$ showed that although pulp $C$ was not so strong as pulp $B$, it was considerably stronger than $A$ and produced a softer paper of better color

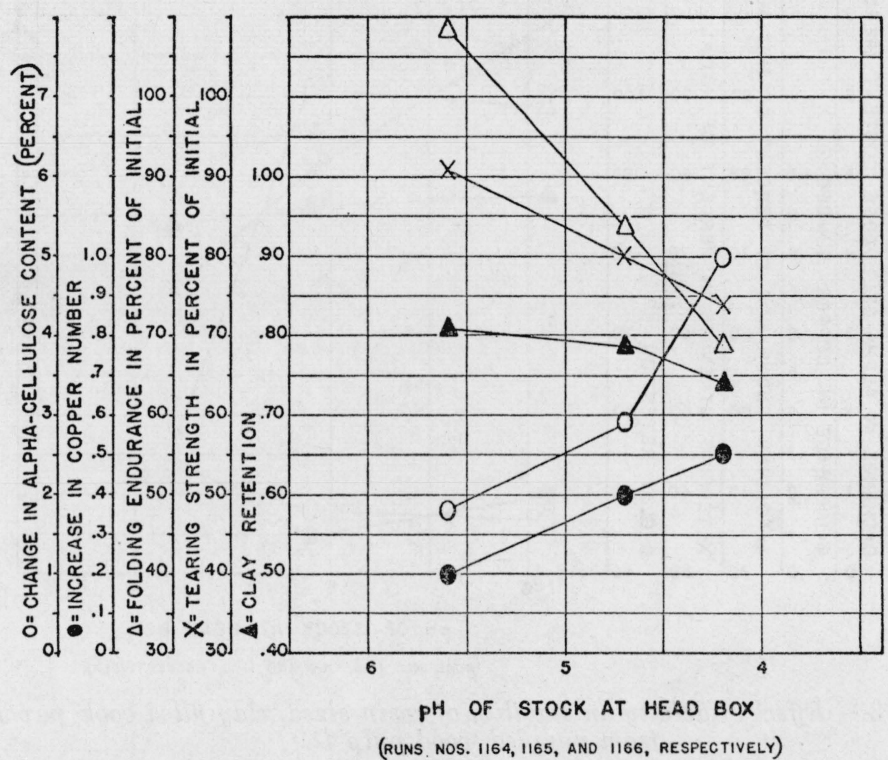

FIGURE 2.-Effect of acidity on stability of rosin-sized, clay-filled book papers made from purified wood pulps $A$ and $C$.

and stability than $B$. Therefore, pulp $C$, rather than $B$, was selected for mixture with purified wood pulp $A$ in the present study to obtain improved strength.

One run (1160) was made of the mixture of pulps $A$ and $C$ with clay filler but without rosin size or alum to obtain data on the quality of the pulp mixture. To determine the effects of rosin size and alum on the stability of papers from the mixture, two series of runs were made - one (runs 1161 to 1163), with 2 percent of rosin size and various amounts of alum; the other (runs 1164 to 1166), with 1 percent of rosin and various amounts of alum. The data on the papers are given in tables 3 and 4 . The stability of the second series (furnish containing 1 percent of rosin) is also shown graphically in figure 2 .

The stability of the papers to the heat treatment decreased as the amount of alum was increased, and was little affected by rosin size. The clay retention was highest when the $\mathrm{pH}$ at the head box was 5.0 to 6.0 and lowest at the highest acidity, $\mathrm{pH} 4.2$. The papers were of 
good formation, color, and strength, showed little change in color after oven-aging, and compared favorably with those made from new white rags, described herein later.

(3) Purified wood pulp: D, 100 percent.-In previous work [4] paper made from purified wood pulp $D$ was hard, and therefore not suitable for book paper, but some of the hardness was attributed to the beater roll and beater tackle not being suited to produce from hard longfibered pulp the desired character of sheet for book paper. It was believed, however, that if the beating could be effected quickly enough to preclude excessive hydrating or gelatinizing of the fibers without

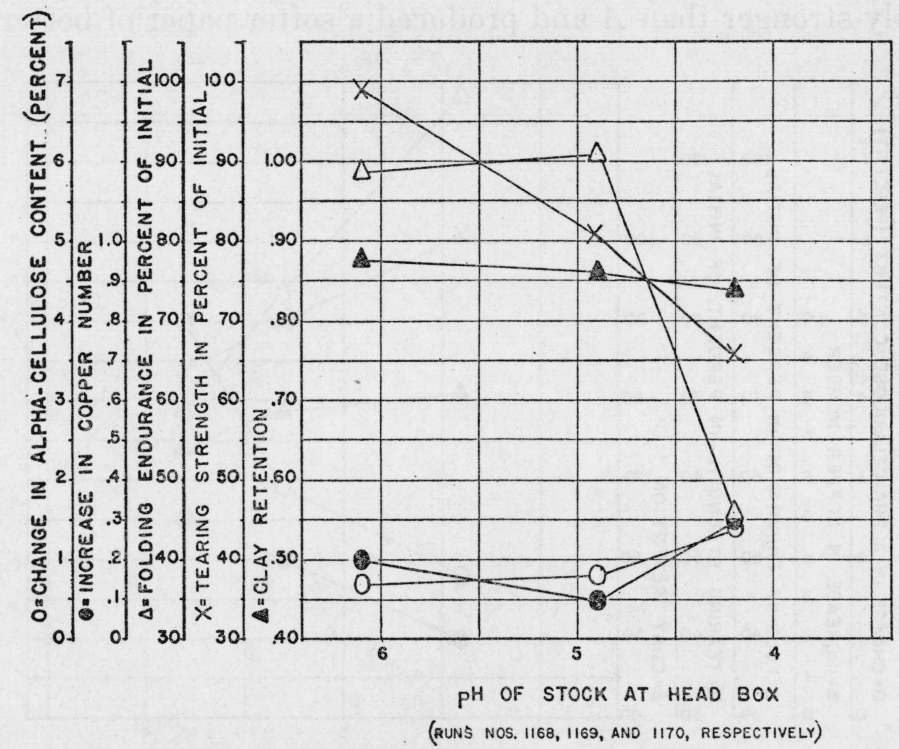

FIGURE 3.-Effect of acidity on stability of rosin-sized, clay-filled book papers made from purified wood pulp D.

sacrificing the desired fraying and fibrillation, a soft bulky sheet would result and would have the strength for severe service. Pulp $D$ was therefore included in the present study.

One paper-machine run (1167) was made from the pulp with clay incorporated in the furnish but without rosin size or alum, and a series of runs (1168 to 1170) using 1 percent of rosin size and various amounts of alum. The stability of the sized papers is shown graphically in figure 3.

The papers showed less deterioration in the heat treatment than some of the other pulps, and better clay retention, but the retention decreased as the acidity was increased. Perhaps because of the unfavorable beating conditions, the alum affected the hydrating or binding properties developed in the beater, thereby reducing the slowness of the stock and the amount of filler retained mechanically. As would be expected, the opacity was low and the paper was comparatively hard-more like writing than book.

(4) Rags: No. 1 new whites, 100 percent.-Three series of papers were made from new rags and clay filler. One series (runs 1176 to 
1178) was without rosin size but with various amounts of alum; the second (runs 1179 to 1182 ) contained 2 percent of rosin size and various amounts of alum; and the third (runs 1183 to 1186), 1 percent of rosin size and various amounts of alum.

The preparation of the rag half stuff and the method of beating the half stuff to prepare it for the paper machine followed the procedure described in previous publications $[3,4]$. When beating the furnish for the rosin-sized papers, however, the stock became very hot as a result of hot weather and hard beating. To preclude any harmful influence of the high temperature on the sizing effect of the rosin, part of the stock was emptied into the chest after the beating was completed so that water could be added to the remainder in the beater before the rosin size was put in. After the rosin size was added, the

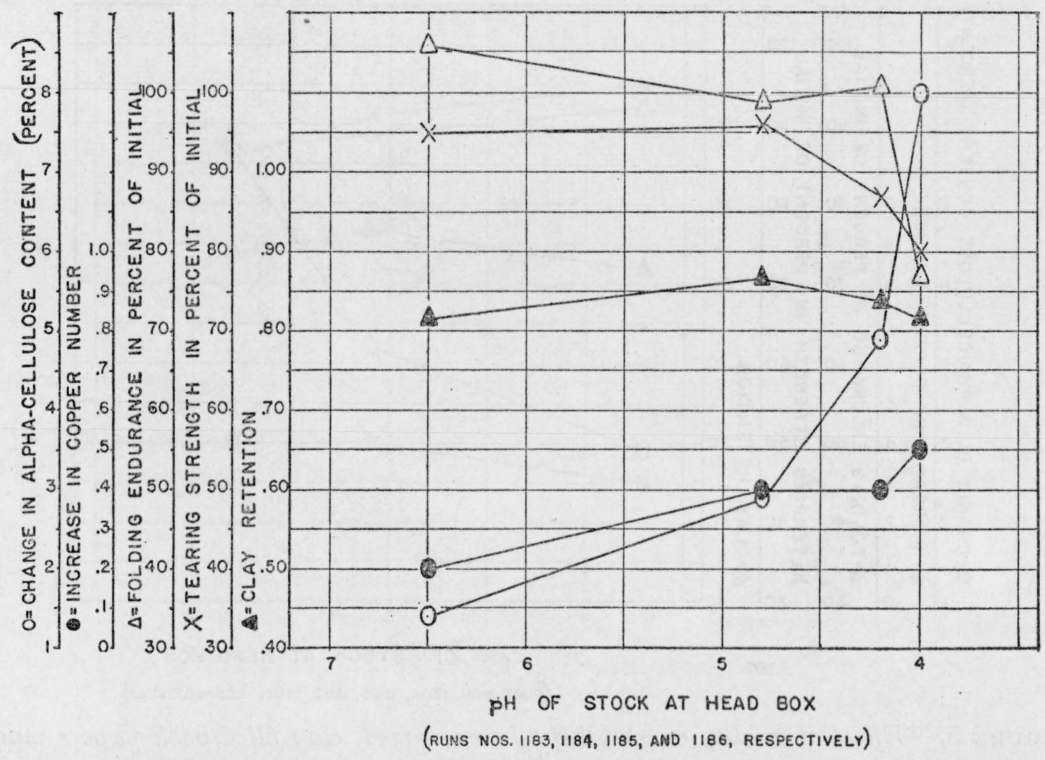

FIGURE 4.-Effect of acidity on stability of rosin-sized, clay-filled book papers made from new rags.

stock was circulated about 15 minutes (with the beater roll off the bedplate), the alum was added, and the stock finally mixed in the beater chest.

The folding endurance of the original papers decreased as the alum was increased, the decrease being greatest for the series with the highest rosin content. The unsized papers showed practically no reaction to the heat treatment in retention of folding endurance and tearing strength, and the rosin-sized papers decreased only slightly. An additional run in each of the sized series was made with the amount of alum increased to 4 percent. The resultant papers also were fairly stable. As is apparent from the test data of tables 3 and 4 and the curves of figure 4, if new white rags are properly prepared and the amounts of rosin size and alum added are not excessive, book paper of high stability can be produced. 
The papers were not well sized but were satisfactory in this respect for printing. The color, formation, and finish were very good. The retention of clay was maximum when the $\mathrm{pH}$ of the stock at the head box was about 5.0. Also, at that acidity the tendency to foam was minimum, not only for the rag stocks but also for the other pulps when rosin size and alum were added.

(5) Rags: No. 1 old whites, 50 percent; twos and blues, 50 percent.The preparation of the rag half stuff from No. 1 old whites and twos and blues also was the same as that used in previous studies [3, 4]. The half stuffs from the two kinds of rags were kept separate until blended in the beater at the time of furnishing. Three series of runs, comparable to the runs described for the preceding pulps in respect of the amounts of clay, rosin, and alum added, were made. One run

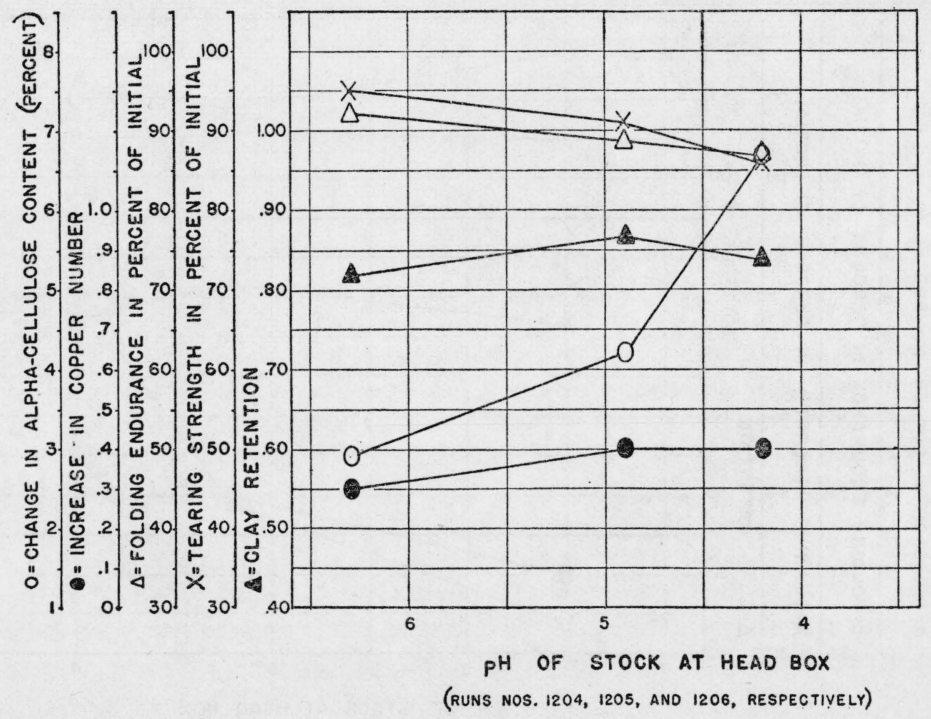

FIGURE 5.-Effect of acidity on stability of rosin-sized, clay-filled book papers made from old rags.

(1214) without clay filler but with rosin size and alum was also included.

The first two runs of old-rag papers (1191 and 1192) without rosin size but with alum showed little change in physical and chemical tests after heat treatment, but the paper of run 1193 , with $\mathrm{pH} 4.2$ at the head box, showed a decided decrease in alpha-cellulose content and retention of folding endurance. The clay retention was practically the same for all three runs.

For both series of the rosin-sized papers ( 2 percent, runs 1200 to 1203 ; 1 percent, runs 1204 to 1206 ) the measurements given in table 4 show increases in the change of alpha-cellulose and copper number as the alum was increased, but the initial folding endurance (table 3) of the papers was so low that the decrease for the heat-treated samples was not pronounced. Clay retention seems to be highest for the old-rag papers when the $\mathrm{pH}$ value at the head box is approximately 5 . Characteristics of the series sized with 1 percent of rosin are shown in figure 5. 


\section{(b) TITANIUM-DIOXIDE-PIGMENTED PAPERS}

Titanium dioxide pigments are manufactured pigments said to produce a high degree of opacity and brightness in paper. Although the manufacturer reported that only small proportions of titanium dioxide pigments are generally used in paper, since our study was concerned mainly with the effect of the pigments on stability, 5 and 15 percent were each used in the experimental runs. The method of furnishing the beater was the same for the titanium dioxide pigments as for the clay filler.

(1) Sulfite pulp, 50 percent; soda pulp, 50 percent.-Two papermachine runs (1147 and 1145) were made with 15 and 5 percent of titanium dioxide pigment $A$, at $\mathrm{pH}$ values 4.8 and 5.0 , respectively, at the head box; and two runs (1148 and 1149) with 15 percent of titanium dioxide pigment $B$ but with different amounts of alum. The beater furnishes all contained 1 percent of rosin size.

The opacity of the paper for which 5 percent of titanium dioxide pigment $A$ was added in the beater was equal to that of the runs in which 15 percent of clay was used; and when 15 percent of titanium dioxide pigment, $A$ or $B$, was used the opacity was better. The papers containing titanium dioxide pigments were whiter and brighter than the clay-filled sheets. The relative quality of the clay and of the other fillers should be regarded as applying only to materials that were representative at the time the work was done. The relationship may be changed with further improvement of fillers. The sizing values were not affected by the pigments. The stability of the titanium-dioxide-pigmented papers was about the same as that of the clay-filled papers.

The original folding endurance and tearing strength of the paper in which 5 percent of pigment $A$ (run 1145) was used were higher than when 15 percent of clay or titanium dioxide pigment was used. To maintain high strength and at the same time obtain high opacity is a result desired in filled papers. Since strength is adversely affected as the amount of filler is increased, relatively high opacifying power is a very desirable property. The retention of titanium dioxide pigment $B$ increased as the amount of alum added was increased.

(2) Rags: No. 1 old whites, 50 percent; twos and blues, 50 percent.Runs comparable to those made from the sulfite-soda pulps were made from old rags also. The stock for one (run 1207) contained 5 percent of titanium dioxide pigment $A$ and was at $\mathrm{pH} 4.9$ at the head box; for two runs (1208 and 1209) the furnishes included 15 percent of titanium dioxide pigment $B$ and varied amounts of alum. All contained 1 percent of rosin size.

As with the sulfite-soda papers, the opacity of the paper (run 1207) for which 5 percent of titanium dioxide pigment $A$ was used was as high as that of the papers in which 15 percent of clay was used, and was higher for the runs containing 15 percent of pigment $B$. The finished titanium-dioxide-pigmented papers also were whiter and brighter. The effect of the titanium dioxide pigments on the stability of the papers, and the degree of retention of the pigments, were about the same as for clay.

\section{(c) ZINC-SULFIDE-PIGMENTED PAPERS}

Zinc sulfide pigments are manufactured materials said to have high brightening and opacifying value. 
The supplier of the zinc sulfide pigments recommends as a precautionary measure that plants having considerable copper equipment "avoid acid conditions so excessive as to attack this equipment and to form a dilute copper solution, as such a condition can dull down the white pigment to an extent dependent upon the amount of copper in solution and the time available for the reaction." The 50-pound beater used at the Bureau is lined with copper, and the stock pipes and screen plates are brass. Since the program of study included the manufacture at $\mathrm{pH} 4.2$ of some papers with each filler, dulling of the papers pigmented with zinc sulfide was expected.

The method of furnishing the beater was the same as that used with clay and with titanium dioxide pigments. The papers were all sized with 1 percent of rosin.

(1) Sulfite pulp, 50 percent; soda pulp, 50 percent.--Paper machine runs 1150 and 1151 were made with 15 and 5 percent of zinc sulfide pigment $A$, at $\mathrm{pH} 4.9$ and 5.0, respectively, at the head box; runs 1152 and 1153 , with 15 percent of zinc sulfide pigment $B$ and various amounts of alum.

All four papers were darkened somewhat, but this condition may have been due to the copper and brass of the equipment and the degree of acidity of the stock. (The odor of hydrogen sulfide was detected at the higher acidities, lower $\mathrm{pH}$ 's.) As a result the opacity would naturally be slightly higher because relatively more light would be absorbed than if the papers had been whiter and brighter in color. The opacity for the run using 5 percent of zinc sulfide pigment $A$ is as high as that of papers made with 15 percent of clay, and the opacities for the runs with 15 percent of zinc sulfide pigments, $A$ or $B$, are higher.

The stability of the zinc-sulfide-pigmented papers was as good as of the papers containing the other fillers. The usual copper number test as a measure of degradation is not applicable to papers containing zinc sulfide because it interferes with the chemical reactions in the test; therefore no values are given in the table. The retention of zinc sulfide pigment $B$ increased as the proportion of alum in the furnish was increased.

(2) Rags: No. 1 old whites, 50 percent; twos and blues, 50 percent.Runs comparable to the last three sulfite-soda runs with zinc sulfide pigments were made with old-rag half stuff also. The test data indicate that as to stability the zinc sulfide pigments had no harmful effect on the papers. But the papers were discolored, which could be attributed in part at least to the copper and brass equipment and the degree of acidity.

\section{(d) CALCIUM-CARBONATE-PIGMENTED PAPERS}

Two types of calcium carbonate pigments were used in this study, precipitated and water-ground natural material. When the work was begun only two samples were considered, one of each kind, the precipitated and sample $A$ of the natural. Later two more samples, $B$ and $C$, of the water-ground natural calcium carbonate were added. The producer of the water-ground natural pigments stated that the three samples differed only in fineness: "Sample $A$, average particle size 10 microns, nothing larger than 30 microns; sample $B$, average particle size 7 microns, nothing larger than 20 microns; sample $C$, average particle size 2 microns, nothing larger than 7 microns." 
The precipitated calcium carbonate was soft and bulky, was more finely divided and more uniform in particle size, and when mixed with water stayed in suspension for a comparatively long time. The waterground natural material was not so soft nor so bulky as the precipitated, and settled out of the water mixture more rapidly. The analyses (table 2) of the two kinds of calcium carbonate show them to be about the same chemically, and they were approximately alike in color.

Most book paper in which alkaline fillers are used is not sized, has no acidic material added, and consequently the stock is alkaline during its manufacture into paper. The general manufacturing practice is very much the same as for the usual clay-filled sheet except for the omission of size and alum. In the experimental work at the Bureau some of the calcium carbonate papers were made without sizing materials, but the pulp for some was rosin-sized first and the calcium carbonate was added later. Before calcium carbonate is used for commercial paper manufacture, however, the patent rights on the use of the material in sized papers should be examined.

(1) Sulfite pulp, 50 percent; soda pulp, 50 percent.-In preparing the sulfite-soda stock for the papermaking runs the pulp mixture was beaten first, and the subsequent operations depended on whether the stock was to be rosin sized. The procedure followed for each run is described under the discussion of the run.

The precipitated calcium carbonate was used in runs 1158 and 1159 and 1172 and 1173 . In run 1158 the calcium carbonate slip was added when the stock was being discharged into the beater chest. No rosin size or alum was added.

The pulp for run 1159 was sized in the beater and the calcium carbonate was added later in the machine chest. Two percent of size was used, based on the weight of pulp and calcium carbonate, or 2.3 percent if based on pulp alone. The sized stock was allowed to stand overnight in the beater chest. The $\mathrm{pH}$ of the stock, before the calcium carbonate was added, was 5.0. The calcium carbonate was added 1 hour before the stock was run over the paper machine.

Run 1172 duplicated run 1159 except for the interval between the addition of the rosin size and the calcium carbonate. For run 1172 the rosin size was added to the pulp in the beater 1 hour before it was discharged into the chest and $1 / 2$ hour before the alum was added. After being emptied into the beater chest the stock was agitated for a short time and then pumped to the machine chest. The $\mathrm{pH}$ of the stock in the beater chest was 5.0. The calcium carbonate slip was added in the machine chest and the stock was agitated for 1 hour to insure uniformity of the mixture. The $\mathrm{pH}$ of the stock at the head box was 8.0.

Run 1173 was similar to run 1172 , except that 30 percent of precipitated calcium carbonate was added.

The water-ground natural calcium carbonate samples $A$ and $C$ were used with the sulfite and soda pulp mixture. The stock for run 1174 was prepared without rosin size or alum, but 30 percent of calcium carbonate sample $A$ was added in the machine chest. The stock was then agitated for 1 hour to insure a uniform mixture for the paper machine.

For run 1175 rosin size was added to the pulp in the beater 1 hour before the stock was emptied into the beater chest and $1 / 2$ hour before the alum was put in. After being discharged to the beater chest 
the stock was agitated for a short time and then pumped to the machine chest. The $\mathrm{pH}$ of the stock in the beater chest was 5.0. Fifteen percent of natural calcium carbonate sample $A$ was added in the machine chest, after which the stock was agitated for 1 hour and then pumped to the paper machine. The $\mathrm{pH}$ of the stock at the head box was 7.7 .

Run 1220 differed from run 1175 only in that 30 percent of calcium carbonate sample $C$ instead of 15 percent of sample $A$ was added.

Natural calcium carbonate sample $A$ (runs 1174 and 1175) settled out of the stock somewhat in the riffler, or sand trap, while being run to the paper machine. This condition was not observed when either the precipitated calcium carbonate or the more finely ground natural calcium carbonate sample $C$ was used.

The stock containing calcium carbonate, precipitated or natural, but no size nor alum, did not foam on the paper machine. When rosin size and alum had been added in the beater, however, followed by calcium carbonate in the machine chest, there was foaming on the paper machine, more for the precipitated than for the natural samples, although the amount was not great and doubtless could have been kept down satisfactorily with a fine water spray. There is, of course, always the possibility when foaming has occurred of foam spots being left in the finished paper.

The sizing values reported in table 3 for the sized papers are not high, but appraised by personal opinions and judgment the papers were sized sufficiently to be written on with ink and for ordinary printing processes. There is no direct correlation between the resistance of paper to water penetration and its ink-receptiveness. The retention of the calcium carbonate and the opacity of the papers were good.

From the physical and chemical test data it appears that papers containing calcium carbonate are more stable than the usual rosinsized papers, which are acid in character.

(2) Rags: No. 1 old whites, 50 percent; twos and blues, 50 percent.In the runs with old rags the calcium carbonate slip was added to the stock in the machine chest and the resultant mixture was agitated for 1 hour before being pumped to the paper machine.

Precipitated calcium carbonate, 15 and 30 percent, respectively, was used in runs 1215 and 1216.

The three paper-machine runs (1217 to 1219) with water-ground natural calcium carbonate comprised 15 percent of samples $C, B$, and $A$, respectively. As previously stated, the only difference in the three samples was the fineness to which they had been ground, $A$ being the coarsest and $C$ the finest. In run 1219, as in the sulfitesoda runs with sample $A$, some of the carbonate settled out from the stock in the riffler, or sand trap, but samples $B$ and $C$ (runs 1218 and 1217) seemed to remain in suspension.

There was no foaming of the stock on the paper machine in any of the runs. The finish of the papers containing the carbonates was satisfactory. All the carbonate-filled papers were comparatively stable.

\section{PRINTING TESTS}

In the early part of the study, printing tests were made at the Government Printing Office on the experimental papers that had 
been manufactured up to that time. The papers printed were representative samples of sulfite-soda runs containing clay (unsized and sized sheets), titanium dioxide pigment $A$, zinc sulfide pigment $A$, and calcium carbonate (precipitated and natural); and of purifiedwood-fiber and new-rag papers containing clay.

The papers were printed from type on one side and by the offset process on the other. The fillers seemed well anchored to the fiber and did not dust out during printing. The papers caused no trouble in the operations and the printings were considered very satisfactory.

Now that the experimental paper-mill work on the book papers has been completed, extensive printing tests to evaluate the printing quality of papers representative of all the different pulp and filler furnishes is in progress and the results obtained will probably appear in a later publication. Final opinion as to the relative values of the different fillers and of the other different papermaking details should await the results of the printings.

\section{DISCUSSION AND CONCLUSIONS}

It is well known that fillers used in large amounts very appreciably reduce the strength of paper. The strength of the experimental papers decreased with increasing filler content, but was influenced by the amount, not the type, of filler present. Because of their effective opacifying quality, smaller amounts of titanium dioxide and zinc sulfide pigments than of clay were sufficient to obtain requisite opacity for printing processes, and the resulting papers were less reduced in strength. All the papers had sufficient strength to withstand the mechanical stresses to which book papers are ordinarily subjected.

There was no pronounced difference in the relative effect of the nonalkaline fillers on sizing. The degree of sizing was very much greater for the sulfite-soda papers than for the rag papers, and the purified wood-fiber papers were in an intermediate position. The nonalkaline fillers had less effect than calcium carbonate in reducing sizing. Although the sizing values of the carbonate papers were not high the papers were sized sufficiently to be written on with ink and for ordinary printing processes. There was no direct correlation between the resistance of the papers to water penetration and their ink-receptiveness.

When rosin size and alum had been added to the stock in the beater and followed by calcium carbonate in the machine chest, the stock foamed somewhat on the paper machine, although the amount of foam was not great and doubtless could have been kept down satisfactorily with a fine water spray.

Maxium clay retention was obtained in the purified wood and rag papers when the $\mathrm{pH}$ at the head box was approximately 5 , and decreased as the amount of alum was increased. For the sulfite-soda pulp, retention of all the nonalkaline fillers increased as alum was increased. Retention of the calcium carbonate was satisfactory.

The papers containing titanium dioxide, zinc sulfide, or precipitated calcium carbonate pigments had the highest opacity values in the experimental work, and, by personal judgment, gave the best printing: results. Only a few of the papers were submitted to the printing tests however. Further tests of the printability of representative samples 
of all the papers are in progress, and rating of their printing qualities will probably be reported in a subsequent publication.

Aside from natural aging the best information on the inherent permanence of paper is based on changes in physical and chemical characteristics during accelerated aging tests. The Bureau believes that oven aging rapidly accelerates the slow deterioration caused by impurities in the paper, and that changes in alpha-cellulose content and copper number and the percentage of the original strength retained indicate the comparative resistance to degradation. The change in the cellulose in the accelerated aging seemed to have been from alpha- to beta-cellulose, with no appreciable difference in the percentage of gamma-cellulose. The rag and purified wood-pulp papers were more stable to the heat test than the sulfite-soda wood-pulp papers. Nonalkaline fillers had no apparent influence on the stability of any of the papers, and the calcium carbonate pigments had a protective or inhibiting effect in the accelerated aging.

Acidity was an important factor in deterioration. Attack on the cellulose was increased as the amount of alum was increased, in either the unsized or the rosin-sized papers. The rag fibers seemed to withstand acidity better than any of the other fibers used. Contrary to the reaction with the pulps of higher initial purity, sulfite-soda papers were more stable to the heat test when containing rosin sizing than when made with corresponding acidity but without size.

The effect on the stability of increasing the amount of alum in the beater and then neutralizing part of the alum with sodium carbonate as the stock was being pumped from the beater chest to the machine chest was practically the same as having had the final $\mathrm{pH}$ value originally in the beater and maintained throughout the preparation of the stock.

The necessity for employing only minimum amounts of rosin and alum in the sizing of papers intended to be used for permanent records is generally recognized. The optimum $\mathrm{pH}$ value for combining high stability with adequate sizing of papers containing nonalkaline fillers, however, varies in different mills because of hardness of water, whitewater recovery, kinds of materials used, etc., but at the Bureau is approximately 5 at the head box of the paper machine. The $\mathrm{pH}$ (hotwater extraction) of the finished papers is in approximate agreement with that of the stock at the head box.

Resistance of paper to deterioration from internal causes is not sufficient to insure its stability, however. The conditions under which the paper is stored and used must also be considered. For a discussion of external deteriorative agencies-light, temperature, humidity, acidic pollution of air-and recommendations as to storage conditions for prolonging the life of paper, the reader is referred to a previous Bureau publication [9].

The authors acknowledge their indebtedness to R. W. Carr of this Bureau for the physical testing of the experimental papers, and to H. F. Launer, C. I. Pope, and W. K. Wilson, also members of the Bureau staff, for the chemical testing of the pulps and papers. 


\section{REFERENCES}

[1] R. H. Rasch, M. B. Shaw, and G. W. Bicking, Highly purified wood fibers as papermaking material, BS J. Research 7,765 (1931) RP372.

[2] R. H. Rasch, M. B. Shaw, and G. W. Bicking, A study of some factors influencing the strength and stability of experimental papers made from two different sulphite pulps, BS J. Research 11, 7 (1933) RP574.

[3] M. B. Shaw, G. W. Bicking, and M. J. O'Leary, A study of the relation of some properties of cotton rags to the strength and stability of experimental papers made from them, J. Research NBS 14, 649 (1935) RP794.

[4] M. B. Shaw and M. J. O'Leary, Study of the effect of fiber components on the stability of book papers, J. Research NBS 17, 859 (1936) RP949.

[5] M. B. Shaw and G. W. Bicking, Caroá fiber as a papermaking material, Tech. Pap. BS 21, 338 (1927) T340; Further experimental production of currency paper in the Bureau of Standards paper mill, BS J. Research 3, 904 (1929) RP121; Equipment and research work of the Bureau of Standards paper mill, Paper Trade J. 89, 19, 60 (1929).

[6] H. F. Launer, Volumetric determination of alpha-, beta-, and gamma-cellulose in pulps and in papers containing sizing, filler, and other materials, J. Research NBS 20, 87 (1938) RP1068.

[7] R. H. Rasch, Accelerated-aging test for paper, BS J. Research 7, 465 (1931) RP352; Estimating stability of paper by heating, Paper Trade J. 95, 4, 28 (1932); R. H. Rasch and B. W. Scribner, Comparison of natural aging of paper with accelerated aging by heating, BS J. Research 11, 727 (1933) RP620.

[8] M. B. Shaw and G. W. Bicking, Comparison of American and foreign clays as paper fillers, Tech. Pap. 18, 337 (1924) T262; A comparative study of paper fillers, Tech. Pap. 19, 733 (1925) T301.

[9] A. E. Kimberly and B. W. Scribner, Summary report of the Bureau of Standards research on preservation of records, Misc. Pub. BS M154 (1937).

Washington, August 10, 1938. 